\title{
Preliminary evaluation of landings and discards of the Turkish bottom trawl fishery in the northeastern Aegean Sea (eastern Mediterranean)
}

\author{
Çetin Keskin ${ }^{1}$, Francesc Ordines ${ }^{2}$, Celal Ates ${ }^{3}$, Joan Moranta ${ }^{2}$, Enric Massutí ${ }^{2}$ \\ ${ }^{1}$ University of Istanbul, Faculty of Fisheries, Ordu St. 200, 34470 Laleli/Istanbul, Turkey. \\ E-mail: seahorse@istanbul.edu.tr \\ ${ }^{2}$ Instituto Español de Oceanografía, Centre Oceanogràfic de les Balears, Moll de Ponent s/n, 07015 Palma de Mallorca \\ Spain. \\ ${ }^{3}$ University of Muğla Sitkı Koçman, Fisheries Faculty, 48000 Kötekli/ Muğla-Turkey.
}

\begin{abstract}
Summary: This short-term study on commercial discards was carried out in fishing grounds around Gökçeada Island in the northeastern Aegean Sea, a geographic area where fisheries information is rather limited. A total of 28 bottom trawls were performed between 70 and $410 \mathrm{~m}$ depth from December 2009 to February 2010. All the samples were collected on board a commercial trawler under commercial fishing conditions. A total of $3143 \mathrm{~kg}$ of biomass was caught in 28 valid hauls. Landings accounted for about $67 \%$ of the total catch while the remaining $33 \%$ was discarded. Fish species comprised $85 \%$ of the total catch and their composition could be divided into four main groups related to depth: shallow shelf (70 to $88 \mathrm{~m}$ ), deep shelf $(155$ to $180 \mathrm{~m})$, shelf break (196 to $276 \mathrm{~m})$, and upper slope (307 to $410 \mathrm{~m})$. Fish dominated the abundance and biomass of both landings and discards in all bathymetric strata. Echinoderms also accounted for a large proportion of the discards. Mean total catches varied between $78.4 \mathrm{~kg} / \mathrm{h}$ on the shallow shelf and $38.1 \mathrm{~kg} / \mathrm{h}$ on the shelf break with no differences in the landing biomass between groups, but with slightly higher discards over the shallow shelf. Taking into account the preliminary nature of the findings, based on a short-term analysis, the minimum mesh size and minimum landing size currently applied in the Turkish bottom trawl fishery are not sufficient to reduce discards and to protect juveniles of commercially important species in the northeastern Aegean Sea.
\end{abstract}

Keywords: bottom trawl; catch composition; landings; discards; length-frequency; eastern Mediterranean.

Evaluación preliminar de los desembarques y descartes de la pesquería de arrastre de fondo de Turquía en el mar Egeo nororiental (Mediterráneo oriental)

Resumen: Este estudio a corto plazo sobre los descartes de la flota comercial de arrastre se llevó a cabo en las zonas de pesca alrededor de la isla Gökçeada en el noreste del mar Egeo, una zona geográfica en la que la información sobre las pesquerías es bastante limitada. Se realizaron un total de 28 pescas de arrastre de fondo entre 70 y $410 \mathrm{~m}$ de profundidad de diciembre 2009 a febrero de 2010. Todas las muestras se recogieron a bordo de un embarcación de pesca de arrastre siguiendo las mismas condiciones de pesca comercial. Se capturaron un total de $3143 \mathrm{~kg}$ de biomasa en 28 lances válidos. Los desembarques comerciales representaron alrededor del $67 \%$ de la captura total, mientras que el resto $(33 \%)$ se descartó. El $85 \%$ de la captura total estuvo compuesta por especies de peces. La composición específica se pudo separar en cuatro grupos principales relacionados con la profundidad : la plataforma poco profunda $(70$ a $88 \mathrm{~m})$, la plataforma profunda $(155-180 \mathrm{~m})$, el borde inferior de la plataforma $(196$ a $276 \mathrm{~m})$ y el talud superior $(307-410 \mathrm{~m})$. Los peces dominaron la abundancia y biomasa tanto de los desembarques comerciales como de los descartes en todos los estratos batimétricos. Además los equinodermos también fueron un grupo importante de los descartes. La media de las capturas totales varió entre $78.4 \mathrm{~kg} / \mathrm{h}$ en la plataforma poco profunda y $38.1 \mathrm{~kg} / \mathrm{h}$ en el borde de la plataforma, sin diferencias en la biomasa desembarcada entre los dos grupos, pero siendo los descartes ligeramente superiores en la plataforma poco profunda. Teniendo en cuenta el carácter preliminar de los resultados, obtenidos sobre la base de un análisis a corto plazo, el tamaño mínimo de malla de la red y la talla mínima de las especies comerciales aplicada hoy en día en la pesquería de arrastre de fondo de Turquía no son suficientes para reducir los descartes y proteger a los juveniles de las especies de importancia comercial en el Mar Egeo nororiental.

Palabras clave: arrastre de fondo; composición de las capturas; desembarques; descartes; frecuencia de tallas; Mediterráneo Oriental.

Citation/Como citar este artículo: Keskin Ç., Ordines F., Ates C., Moranta J., Massutí E. 2014. Preliminary evaluation of landings and discards of the Turkish bottom trawl fishery in the northeastern Aegean Sea (eastern Mediterranean). Sci. Mar. 78(2): 213-225. doi: http://dx.doi.org/10.3989/scimar.03942.30B

Editor: S. Somarakis.

Received: August 31, 2013 Accepted: February 21, 2014. Published: May 29, 2014. 
Copyright: (C) 2014 CSIC. This is an open-access article distributed under the Creative Commons Attribution-Non Commercial Lisence (by-nc) Spain 3.0.

\section{INTRODUCTION}

During the last few decades, scientific studies have revealed the intensive impact of fishing activities on ecosystems (e.g. Jennings and Kaiser 1998). Among negative effects such as reduced species abundances, changes in size and species composition, and modification of species life-history traits (e.g. Gislason et al. 2000), one of the most undesirable direct effects of fishing is the great amount of by-catch and discards produced all around the world (Kelleher 2005, Davies et al. 2009). The fishing techniques in use in the Mediterranean Sea are highly diverse, but bottom trawling has the greatest ecosystem impact because it involves a wide range of species and different functional groups (Coll et al. 2006, Moranta et al. 2008, Tsagarakis et al. 2013). Discards produced by this fishing activity account for $20 \%$ to $70 \%$ of the total catch depending upon the geographic area, depth range and type of habitat where fishing occurs (Stergiou et al. 1998, Moranta 2000, Machias et al. 2001, 2004, Sánchez et al. 2004, 2007, Guijarro and Massutí 2006, Ordines et al. 2006).

The Aegean Sea is one of the main biogeographic areas of the Mediterranean Sea (Garibaldi and Caddy 1998) and, according to the spatial analysis of catch composition of the commercial fleet operating in this area, two main regions, the northern and southern Aegean, have been defined (Stergiou and Pollard 1994). Available studies have revealed differences in the demersal assemblages between these regions (Tsimenides et al. 1991, Kallianiotis et al. 2000, 2004, Labropoulou and Papaconstantinou 2000, Katsanevakis and Maravelias 2009, Klaoudatos et al. 2010)

Thanks to increased research effort in the southern and northwestern region of the Aegean, information on the demersal fisheries and their impact on ecosystems has improved substantially (e.g. Petrakis and Stergiou 1997, Stergiou et al. 1997, Smith et al. 2000, Labropoulou and Papaconstantinou 2004, Damalas and Vassilopoulou 2011, Maravelias et al. 2012, Katsanevakis et al. 2009, Damalas et al. 2010, Tosunoğlu et al. 2003a, 2003b, Tokaç et al. 1998, 2004). Similarly, demersal fish communities of the northeastern Aegean have also been studied and compared with other areas (Labropoulou and Papaconstantinou 2000, 2004, Keskin et al. 2011a, 2011b). However, the information on catch composition, landings and discards of the bottom trawl fishery in the eastern Aegean Sea is very scarce (Özbilgin et al. 2006).

Demersal species landings by the Turkish fishing fleet operating in the Aegean Sea are on average 10000 t per year (Tirasın and Ünlüoğlu 2012), being about $31 \%$ of its total fisheries production. This is the fourth most important region in fisheries production in Turkey, accounting for nearly $10 \%$ of the total marine fisheries landings. The number of fishing boats in Turkey is around 17803 (TUIK 2009). A total of 5725 of them are located in the Aegean Sea, corresponding to $32 \%$ of the total fleet. Among them 83 are bottom trawlers, 89 purse seiners, 17 trawler-purse seiners (combined) and 5056 small-scale fishing boats.

The current fishing regulations in Turkey include: i) minimum mesh size and landing sizes; ii) closed areas and seasons; iii) gear or fishing method restrictions and bans; and, iv) catch prohibition for some species (Anonymous 2012). Fishing effort is regulated by a system of vessel licensing, which was adopted in 2002. As a part of the European Union (EU) accession process to correct legal, institutional and structural deficiencies, Turkey's fisheries have recently been subject to evaluation and fisheries regulation is currently being revised (Koşar 2009, European Commission 2007). The aim of these reforms is permit a more effective fisheries management. Some new measures have already been implemented (e.g. a total allowable catch for Bluefin tuna and vessel length limitation for some species) and some technical improvements for a future implementation of vessel monitoring and fisheries information systems have been made. However, no new management measures have thus far been implemented for demersal fisheries (Anonymous 2012).

Despite the available information on the Mediterranean bottom trawl fishery, additional scientific studies are still necessary to better understand the dynamics of catches and discards (Piet and Jennings 2005), especially in data-poor areas. It must be taken into account that one of the main objectives of the new Common Fishery Policy of the EU is the gradual elimination of discards from 2014 onwards (European Commission 2011). The main objective of this paper is to improve the knowledge of demersal communities exploited by the bottom trawl fishery off Gökçeada Island (northeastern Aegean Sea), focusing on the bathymetric distribution of species, composition of landings and discards, and sizes of the main commercial fish species.

\section{MATERIALS AND METHODS}

\section{Study area}

The North Aegean Sea is a highly productive area because of the influence of the Black Sea and the Meriç River (Ignatiades et al. 2002). The bottom type on the shelf is mainly sand, with silt and clay sediments found predominantly on the slope (Sarı and Çağatay 2001).

The main fishing grounds in Turkish territorial waters in the Aegean Sea are located in Saros Bay, around Gökçeada and Bozcaada islands, and in Edremit Bay in the north, Çandarlı Bay, İzmir Bay, Sığacık and Kuşadası Bay in the centre, and Güllük Bay and Gökova Bay in the south (Kinacigil and İlkyaz 2012). The study area was located on the fishing grounds around Gökçeada Island (Fig. 1). Fishing activities in this entire region are spatially limited due to the narrow continental shelf, the steep slope and its geo-morphological characteristics (Benli et al. 1999, K1nacıgil and İlkyaz 2012). Due to its composition (vessel sizes range from 15 to $25 \mathrm{~m}$ with an engine size of 150-350 HP) and lack of appropri- 


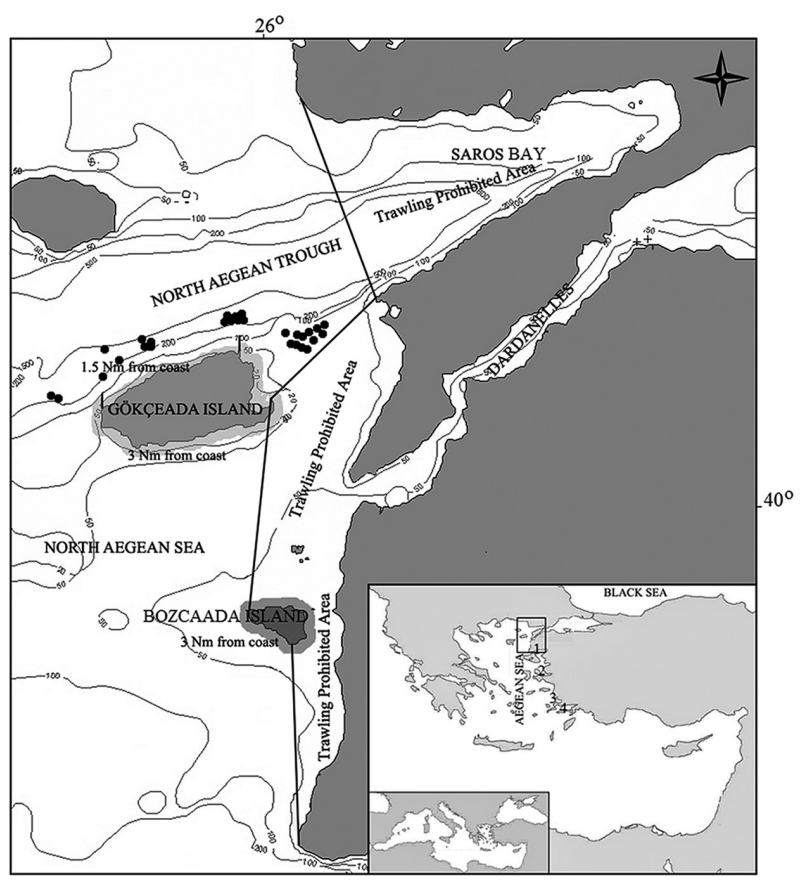

Fig. 1. - Map of the study area around Gökçeada Island (northeastern Aegean Sea; eastern Mediterranean) showing the position of the trawl samples analysed (circles). 1, Edremit Bay; 2, İzmir Bay; 3, Güllük Bay; 4, Gökova Bay.

ate equipment, the bottom fleet is limited to exploiting depths of 400-500 m (Kinacigil and İlkyaz 2012, Tirasin and Ünlüoğlu 2012). The most important target species of the bottom trawl fishery are red mullet Mullus barbatus (L.), European hake Mercluccius merluccius (L.), common sole, Solea solea (L.), pandora (Pagellus spp.), poor cod Trisopterus minutus (L.), whiting Merlangius merlangus (L.), anglerfish Lophius spp., four-spot megrim Lepidorhombus boscii (Risso), Norway lobster Nephrops norvegicus Leach, rose shrimp Parapenaeus longirostris (Lucas) and giant red shrimp Aristeomorpha foliacea (Risso) (Tirasın and Ünlüoğlu 2012).

The fisheries management in the area is based on closed areas and seasons. Bottom trawling activities are banned within 1.5 miles of the north coast and within 3 miles of the south coast off Gökçeada Island to protect small fishes and Posidonia oceanica (L.) Delile meadows. In addition, Saros Bay, a very important area for fish spawning and nursery (Coker et al. 2008), has been closed to trawling since 1993. In Turkish territorial waters, trawling is not allowed from April to September in order to protect the recruitment of demersal fish species. For these reasons, the existing open areas in the northeastern Aegean Sea for bottom trawling are heavily exploited during the fishing season (November to April). Unfortunately, for some areas, such as our study area, there are no reliable data and/or official statistics on fishing effort and the number of vessels operating is unknown. Nevertheless, many bottom trawlers were observed in the area during the sampling period.

\section{Sampling and data analysis}

Sampling was conducted in December 2009 and February 2010 onboard a commercial vessel (24 m length and 141.5 GRT) and under commercial fishing conditions. A conventional bottom trawl gear, with vertical and horizontal opening estimated at around 1.5 and 22 $\mathrm{m}$, respectively, and a codend stretched diamond mesh size of $44 \mathrm{~mm}$ was used. Observers on board collected data from 28 hauls, carried out between 70 and $410 \mathrm{~m}$ depth. The effective duration of these hauls ranged from 1-2 $\mathrm{h}$ on shallow bottoms $(<100 \mathrm{~m})$ to $3-4 \mathrm{~h}$ in deeper fishing grounds. Position, depth and vessel speed were recorded every $30 \mathrm{~min}$. After each haul, catches were sorted by taxonomic and commercial categories (landings and discards), counted and weighed separately. Total length of individuals of commercial fish species was measured to the nearest $\mathrm{cm}$.

Catches in terms of abundance and biomass were standardized to one-hour tows. Species appearing only in one sample were removed from the matrix used for multivariate analysis. Cluster analysis was applied to detect groups of samples with similar species composition in total catches. Data were $\log (\mathrm{x}+1)$ transformed, the Bray-Curtis index was used as similarity index and the UPGM (unweighted pair-group method), with arithmetic mean was applied to link samples into clusters. Similarity percentage analysis (SIMPER) was used to assess species contribution to the detected groups of samples. All analyses were carried out using PRIMER software (Clarke and Warwick 2001). For each group detected in the cluster analysis, mean biomass of total catch, landings and discards, and the community descriptors mean species richness (S), Shannon-Wiener diversity (H'; Shannon and Weaver 1949) and evenness indices ( $J$ '; Pielou 1969) were determined for fish assemblages. Differences in these parameters were tested using one-way ANOVA. Significant differences among groups were identified using the HSD post-hoc test. Data was checked for the assumptions of homogeneity of variances and normality before applying ANOVA. When these assumptions were not met a more conservative $\mathrm{p}$-value $(<0.01)$ was adopted.

\section{RESULTS}

A total of $3143 \mathrm{~kg}$ of biomass were caught in the 28 valid hauls. Of that amount, $2101 \mathrm{~kg}(67 \%)$ were landed and $1042 \mathrm{~kg}$ (33\%) were discarded. Eightynine fish species comprised $85 \%$ of the total catch, and echinoderms (7\%), crustaceans (5\%) and cephalopods (2\%) were also large taxonomic groups (Table 1). Eight fish species were always landed, 49 were always discarded, and 32 appeared in both the landed and the discarded fractions.

The cluster analysis showed the existence of four groups of samples at around $50 \%$ similarity, with the following depth ranges: $70-88 \mathrm{~m}$ (shallow shelf, SS), 155-180 m (deep shelf, DS), 196-276 m (shelf break, $\mathrm{SB}$ ) and 307-410 m (upper slope, US) (Fig. 2). According to SIMPER analysis, the average similarities of the four groups were $70.0 \%$ in the SS group, $70.5 \%$ in the DS group, $66.3 \%$ in the SB group and $80.4 \%$ in the US group. The contribution of the main fish species to the average similarity per each group is shown in Table 2. 
Table 1. - General summary of the results for the trawl samples analysed. Cephal., cephalopods; Crustac., crustaceans; Echin., echinoderms.

\begin{tabular}{|c|c|c|c|c|c|c|c|c|c|c|c|c|c|}
\hline Hauls & Date & $\begin{array}{c}\text { Tow } \\
\text { time } \\
\text { (h) }\end{array}$ & $\begin{array}{l}\text { Min. } \\
\text { depth } \\
(\mathrm{m})\end{array}$ & $\begin{array}{c}\text { Max. } \\
\text { depth } \\
\text { (m) }\end{array}$ & $\begin{array}{c}\text { Mean } \\
\text { depth } \\
(\mathrm{m})\end{array}$ & $\begin{array}{c}\text { Total } \\
\text { catch } \\
(\mathrm{kg})\end{array}$ & $\begin{array}{l}\text { Landings } \\
\quad(\mathrm{kg})\end{array}$ & $\begin{array}{l}\text { Discards } \\
\quad(\mathrm{kg})\end{array}$ & $\begin{array}{c}\text { Fishes } \\
(\mathrm{kg})\end{array}$ & $\begin{array}{l}\text { Cephal. } \\
\text { (kg) }\end{array}$ & $\begin{array}{l}\text { Crustac. } \\
\quad(\mathrm{kg})\end{array}$ & $\begin{array}{c}\text { Echin. } \\
(\mathrm{kg})\end{array}$ & $\begin{array}{c}\text { Others } \\
(\mathrm{kg})\end{array}$ \\
\hline 1 & 05.12 .2009 & 4 & 201 & 233 & 223 & 129 & 94 & 35 & 106 & 3 & 19 & 2 & \\
\hline 2 & 05.12 .2009 & 5 & 263 & 275 & 266 & 185 & 148 & 37 & 174 & 3 & 7 & $\overline{1}$ & \\
\hline 3 & 07.12 .2009 & 4 & 165 & 210 & 196 & 180 & 141 & 39 & 160 & 5 & 12 & 3 & \\
\hline 4 & 07.12 .2009 & 3 & 284 & 323 & 307 & 101 & 68 & 33 & 87 & & 8 & 6 & \\
\hline 5 & 07.12 .2009 & 4 & 262 & 243 & 225 & 105 & 67 & 38 & 90 & 4 & 1 & 9 & 1 \\
\hline 6 & 08.12 .2009 & 4 & 161 & 215 & 196 & 85 & 69 & 17 & 63 & 3 & 17 & 3 & \\
\hline 7 & 08.12 .2009 & 3 & 255 & 270 & 267 & 64 & 59 & 5 & 48 & 2 & 14 & 1 & \\
\hline 8 & 08.12 .2009 & 2 & 133 & 190 & 155 & 110 & 84 & 26 & 84 & 6 & 16 & 4 & \\
\hline 9 & 09.12 .2009 & 3 & 374 & 456 & 410 & 173 & 149 & 24 & 160 & 3 & 10 & 1 & \\
\hline 10 & 09.12 .2009 & 3 & 333 & 449 & 388 & 173 & 137 & 36 & 149 & 7 & 12 & 6 & \\
\hline 11 & 09.12 .2009 & 2 & 343 & 407 & 369 & 49 & 29 & 21 & 44 & & 5 & 1 & \\
\hline 12 & 24.02 .2010 & 3 & 140 & 198 & 168 & 144 & 101 & 43 & 120 & 4 & 8 & 12 & \\
\hline 13 & 24.02 .2010 & 3 & 161 & 205 & 176 & 134 & 91 & 43 & 101 & 4 & 10 & 13 & 7 \\
\hline 14 & 24.02 .2010 & 3 & 123 & 247 & 180 & 132 & 79 & 53 & 95 & 2 & 8 & 27 & \\
\hline 15 & 25.02 .2010 & 3 & 274 & 277 & 275 & 186 & 96 & 90 & 165 & 4 & 8 & 9 & \\
\hline 16 & 25.02 .2010 & 3 & 272 & 279 & 276 & 70 & 51 & 20 & 59 & 1 & 5 & 4 & 1 \\
\hline 17 & 27.02 .2010 & 2 & 83 & 88 & 86 & 70 & 45 & 25 & 64 & 2 & & 4 & \\
\hline 18 & 27.02 .2010 & 1 & 69 & 72 & 70 & 55 & 26 & 29 & 54 & 1 & & & \\
\hline 19 & 27.02 .2010 & 1 & 70 & 73 & 71 & 82 & 32 & 50 & 55 & 2 & & 24 & \\
\hline 20 & 27.02 .2010 & 1 & 79 & 83 & 82 & 53 & 24 & 29 & 42 & 3 & & 7 & 2 \\
\hline 21 & 27.02 .2010 & 1 & 77 & 83 & 81 & 102 & 57 & 45 & 92 & 3 & 2 & 4 & 1 \\
\hline 22 & 27.02 .2010 & 1 & 87 & 89 & 88 & 64 & 40 & 24 & 60 & 3 & & 1 & 1 \\
\hline 23 & 27.02 .2010 & 1 & 74 & 78 & 76 & 65 & 42 & 23 & 59 & 1 & & 2 & 3 \\
\hline 24 & 27.02 .2010 & 1 & 80 & 83 & 81 & 76 & 42 & 34 & 51 & 3 & 2 & 20 & 2 \\
\hline 25 & 27.02.2010 & 1 & 84 & 89 & 86 & 125 & 78 & 48 & 105 & 2 & 1 & 17 & 1 \\
\hline 26 & 27.02 .2010 & 1 & 84 & 91 & 87 & 139 & 61 & 78 & 136 & 2 & & & 1 \\
\hline 27 & 27.02.2010 & 1 & 79 & 83 & 81 & 167 & 110 & 57 & 150 & 2 & & 14 & 1 \\
\hline 28 & 27.02 .2010 & 1 & 81 & 83 & 81 & 123 & 83 & 40 & 96 & 1 & & 24 & 1 \\
\hline Total (kg) & & & & & & 3143 & 2101 & 1042 & 2667 & 76 & 163 & 216 & 22 \\
\hline Percentage & & & & & & & 67 & 33 & 85 & 2 & 5 & 7 & 1 \\
\hline
\end{tabular}

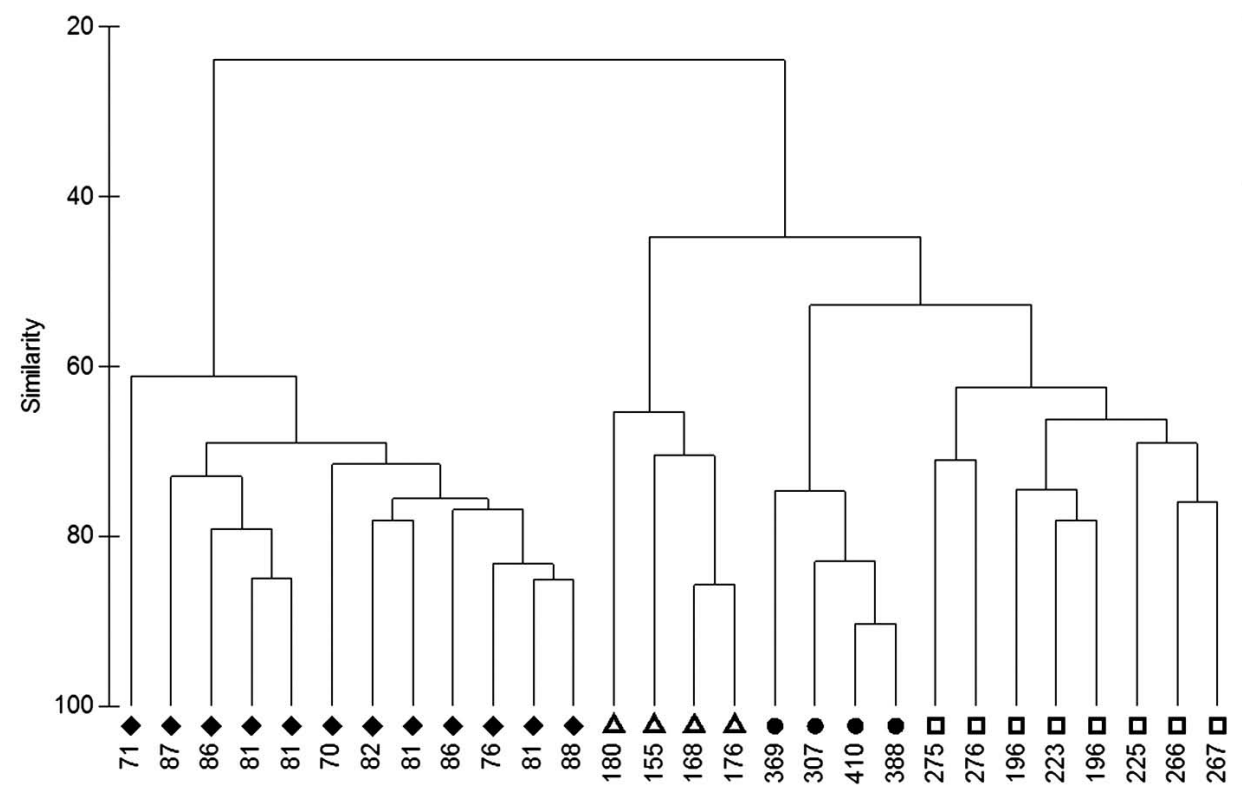

Fig. 2. - Cluster analysis of the species abundance (individuals/h) in the trawl samples . Mean depth is shown for each sample. SS, shallow shelf; DS, deep shelf ; SB, shelf break; US, upper slope.

ANOVA showed no differences in $\mathrm{S}$ among these groups, whereas significant differences were detected for $H^{\prime}$ and $J^{\prime}$, due to higher values on the SS and the US than on the DS and the SB (Table 3). Mean total catches varied between $78.4 \mathrm{~kg} / \mathrm{h}$ on the SS and 38.1 $\mathrm{kg} / \mathrm{h}$ on the SB. Landings biomass showed no differences between groups, whereas the amount of discards was significantly higher on the SS than on the rest of depth strata (Table 3).
Total biomass of both landings and discards was mainly composed of fish species in all groups (Fig. 3). Fish accounted for $96 \%$ of landings in the SS, with $M$. barbatus, Dentex maroccanus Valenciennes, Pagellus acarne (Risso), M. merluccius and P. erythrinus comprising up to $70 \%$ of the commercialized catch (Table $4)$. In the DS, fishes accounted for $88 \%$ of the landings, M. merluccius and $P$. bogaraveo being the most important species (both reaching 72\% of landings). 
Table 2. - SIMPER results for each fish assemblage identified from cluster analysis of hauls. N, abundance (individual/h); Sim, average similarity; SD, Standard deviation; Sim\%, percentage contribution to the similarity; DISSim, average dissimilarity. Letters between brackets indicate whether the species are considered target (T), low-demand (L) and always discarded (D) species according to market demand.

\begin{tabular}{|c|c|c|c|c|c|}
\hline $\mathrm{SS}(70-88 \mathrm{~m}), \mathrm{Sim}=70.61$ & $\mathrm{~N}$ & Sim & $\mathrm{Sim} / \mathrm{SD}$ & $\operatorname{Sim} \%$ & $\Sigma \operatorname{Sim} \%$ \\
\hline $\begin{array}{l}\text { Mullus barbatus }(\mathrm{T}) \\
\text { Pagellus erytrinus }(\mathrm{L}) \\
\text { Pagellus bogareveo }(\mathrm{L}) \\
\text { Lepidotrigla cavillone }(\mathrm{D}) \\
\text { Boops boops }(\mathrm{L}) \\
\text { Spicara smaris }(\mathrm{L}) \\
\text { Citharus linguatula }(\mathrm{L}) \\
\text { Merluccius merluccius }(\mathrm{T}) \\
\text { Dentex maroccanus }(\mathrm{L}) \\
\text { Pagellus acarne }(\mathrm{L}) \\
\text { Serranus hepatus }(\mathrm{L}) \\
\text { Trachurus trachurus }(\mathrm{L}) \\
\text { Scyliorhinus canicula }(\mathrm{D}) \\
\text { Serranus cabrilla }(\mathrm{L}) \\
\text { Spicara maena }(\mathrm{L})\end{array}$ & $\begin{array}{l}202.10 \\
84.07 \\
293.81 \\
153.83 \\
53.67 \\
51.70 \\
45.75 \\
40.66 \\
97.62 \\
116.31 \\
11.64 \\
13.37 \\
7.30 \\
7.94 \\
5.60\end{array}$ & $\begin{array}{l}7.98 \\
6.84 \\
5.79 \\
5.79 \\
5.60 \\
4.84 \\
4.67 \\
3.66 \\
3.40 \\
3.12 \\
2.76 \\
2.72 \\
2.41 \\
2.39 \\
2.06\end{array}$ & $\begin{array}{l}9.14 \\
7.00 \\
1.41 \\
2.41 \\
6.08 \\
3.48 \\
3.55 \\
1.65 \\
1.30 \\
1.25 \\
2.99 \\
4.05 \\
2.75 \\
1.94 \\
1.75\end{array}$ & $\begin{array}{l}11.30 \\
9.69 \\
8.21 \\
8.20 \\
7.93 \\
6.86 \\
6.62 \\
5.18 \\
4.82 \\
4.42 \\
3.90 \\
3.85 \\
3.41 \\
3.39 \\
2.92\end{array}$ & $\begin{array}{l}11.30 \\
20.98 \\
29.19 \\
37.39 \\
45.32 \\
52.18 \\
58.80 \\
63.98 \\
68.80 \\
73.22 \\
77.12 \\
80.97 \\
84.39 \\
87.78 \\
90.70\end{array}$ \\
\hline DS $(155-180 \mathrm{~m}), \mathrm{Sim}=70.51$ & $\mathrm{~N}$ & Sim & $\mathrm{Sim} / \mathrm{SD}$ & $\operatorname{Sim} \%$ & $\Sigma \operatorname{Sim} \%$ \\
\hline $\begin{array}{l}\text { Merluccius merluccius }(\mathrm{T}) \\
\text { Pagellus bogareveo }(\mathrm{L}) \\
\text { Trachurus trachurus }(\mathrm{L}) \\
\text { Citharus linguatula }(\mathrm{L}) \\
\text { Capros aper }(\mathrm{D}) \\
\text { Trigla lyra }(\mathrm{T}) \\
\text { Micromesistius poutassou }(\mathrm{L}) \\
\text { Mullus barbatus }(\mathrm{T}) \\
\text { Raja clavata }(\mathrm{L}) \\
\text { Helicolenus dactylopterus }(\mathrm{L}) \\
\text { Lophinus budegassa }(\mathrm{T}) \\
\text { Zeus faber }(\mathrm{T}) \\
\text { Scyliorhinus canicula }(\mathrm{D}) \\
\text { Lepidotrigla cavillone (L) } \\
\text { Gadiculus argenteus }(\mathrm{D}) \\
\text { Argentina sphyraena }(\mathrm{D})\end{array}$ & $\begin{array}{l}341.07 \\
156.86 \\
55.59 \\
54.46 \\
14.88 \\
6.75 \\
14.01 \\
13.19 \\
3.44 \\
2.65 \\
2.69 \\
1.37 \\
4.39 \\
1.51 \\
5.80 \\
6.87\end{array}$ & $\begin{array}{l}13.02 \\
11.14 \\
5.22 \\
4.46 \\
4.45 \\
3.94 \\
3.74 \\
3.39 \\
3.37 \\
2.25 \\
2.10 \\
1.64 \\
1.61 \\
1.45 \\
1.34 \\
1.21\end{array}$ & $\begin{array}{l}10.94 \\
28.29 \\
4.49 \\
0.91 \\
1.72 \\
5.27 \\
3.56 \\
0.91 \\
5.79 \\
2.31 \\
9.97 \\
9.99 \\
3.06 \\
0.91 \\
0.76 \\
0.90\end{array}$ & $\begin{array}{l}18.46 \\
15.80 \\
7.41 \\
6.32 \\
6.31 \\
5.58 \\
5.30 \\
4.80 \\
4.78 \\
3.19 \\
2.98 \\
2.33 \\
2.29 \\
2.05 \\
1.90 \\
1.72\end{array}$ & $\begin{array}{l}18.46 \\
34.27 \\
41.67 \\
48.00 \\
54.31 \\
59.89 \\
65.19 \\
69.99 \\
74.78 \\
77.96 \\
80.94 \\
83.27 \\
85.56 \\
87.61 \\
89.50 \\
91.22\end{array}$ \\
\hline SB $(196-276 m), \operatorname{Sim}=66.35$ & $\mathrm{~N}$ & $\operatorname{Sim}$ & $\mathrm{Sim} / \mathrm{SD}$ & $\operatorname{Sim} \%$ & $\Sigma \operatorname{Sim} \%$ \\
\hline $\begin{array}{l}\text { Merluccius merluccius }(\mathrm{T}) \\
\text { Scyliorhinus canicula (D) } \\
\text { Gadiculus argenteus (D) } \\
\text { Phycis blennoides }(\mathrm{T}) \\
\text { Micromesistius poutassou }(\mathrm{L}) \\
\text { Helicolenus dactylopterus }(\mathrm{L}) \\
\text { Pagellus bogareveo }(\mathrm{L}) \\
\text { Lophinus budegassa (T) } \\
\text { Trigla lyra }(\mathrm{T}) \\
\text { Capros aper }(\mathrm{D}) \\
\text { Citharus linguatula (L) } \\
\text { Lepidopus caudatus }(\mathrm{D}) \\
\text { Argentina sphyraena (D) } \\
\text { Conger conger (D) }\end{array}$ & $\begin{array}{l}170.16 \\
24.60 \\
16.51 \\
8.96 \\
5.36 \\
7.88 \\
34.32 \\
3.12 \\
3.20 \\
1.41 \\
2.97 \\
1.28 \\
0.76 \\
1.16\end{array}$ & $\begin{array}{l}15.47 \\
7.10 \\
6.12 \\
6.01 \\
4.74 \\
4.44 \\
4.33 \\
3.34 \\
2.33 \\
1.63 \\
1.53 \\
1.34 \\
1.32 \\
1.19\end{array}$ & $\begin{array}{l}10.44 \\
5.28 \\
2.56 \\
8.66 \\
4.34 \\
3.91 \\
1.76 \\
3.04 \\
1.41 \\
1.21 \\
0.69 \\
0.83 \\
2.97 \\
1.97\end{array}$ & $\begin{array}{l}23.31 \\
10.69 \\
9.22 \\
9.06 \\
7.14 \\
6.69 \\
6.52 \\
5.04 \\
3.51 \\
2.46 \\
2.31 \\
2.02 \\
1.98 \\
1.79\end{array}$ & $\begin{array}{l}23.31 \\
34.00 \\
43.22 \\
52.28 \\
59.42 \\
66.11 \\
72.63 \\
77.67 \\
81.18 \\
83.64 \\
85.94 \\
87.97 \\
89.95 \\
91.74\end{array}$ \\
\hline US $(307-410 \mathrm{~m}), \mathrm{Sim}=80.04$ & $\mathrm{~N}$ & $\operatorname{Sim}$ & $\mathrm{Sim} / \mathrm{SD}$ & $\operatorname{Sim} \%$ & $\Sigma \operatorname{Sim} \%$ \\
\hline $\begin{array}{l}\text { Caelorinchus caelorinchus (D) } \\
\text { Hoplostethus mediterraneus (D) } \\
\text { Phycis blennoides }(\mathrm{T}) \\
\text { Merluccius merluccius (T) } \\
\text { Nezumia aequalis (D) } \\
\text { Scyliorhinus canicula (D) } \\
\text { Lepidorhombus boscii (T) } \\
\text { Micromesistius poutassou (L) } \\
\text { Gadiculus argenteus (D) } \\
\text { Etmopterus spinax (D) } \\
\text { Molva dypterygia (L) } \\
\text { Helicolenus dactylopterus (L) } \\
\text { Pagellus bogareveo (T) } \\
\text { Galeus melastomus (D) }\end{array}$ & $\begin{array}{c}148.73 \\
56.06 \\
33.13 \\
52.29 \\
29.82 \\
15.14 \\
12.89 \\
12.89 \\
13.87 \\
5.97 \\
7.31 \\
5.92 \\
23.93 \\
23.45\end{array}$ & $\begin{array}{l}9.82 \\
8.06 \\
6.86 \\
6.75 \\
6.52 \\
5.08 \\
4.23 \\
4.19 \\
3.87 \\
3.56 \\
3.54 \\
3.28 \\
3.19 \\
3.15\end{array}$ & $\begin{array}{l}11.61 \\
12.26 \\
31.59 \\
19.60 \\
7.51 \\
7.02 \\
4.37 \\
1.36 \\
2.71 \\
16.98 \\
6.92 \\
4.89 \\
1.93 \\
0.91\end{array}$ & $\begin{array}{l}12.27 \\
10.07 \\
8.57 \\
8.43 \\
8.15 \\
6.34 \\
5.28 \\
5.24 \\
4.83 \\
4.45 \\
4.43 \\
4.10 \\
3.98 \\
3.93\end{array}$ & $\begin{array}{l}12.27 \\
22.34 \\
30.91 \\
39.35 \\
47.49 \\
53.83 \\
59.11 \\
64.35 \\
69.19 \\
73.63 \\
78.06 \\
82.16 \\
86.14 \\
90.07\end{array}$ \\
\hline $\begin{array}{l}\text { DS \& SS, DISSim }=63.59 \\
\text { SB \& SS, DISSim }=77.74 \\
\text { SB \& US, DISSim }=47.25 \\
\text { SB \& DS, DISSim }=49.04 \\
\text { US \& SS, DISSim }=85.37 \\
\text { US \& DS, DISSim }=67.65\end{array}$ & & & & & \\
\hline
\end{tabular}


Table 3. - Ecological parameters (mean $\pm \mathrm{SD}$ ) for each group resulting from cluster analysis. $\mathrm{n}$, sampling size; $\mathrm{s}$, total species number; $\mathrm{S}$, mean number species; J', mean evenness index; $H^{\prime}$, mean diversity index. SS, shallow shelf (70-88 m); DS, deep shelf (155-180 m); SB, shelf break (196-276 m); US, upper slope (307-410 m). D, discards (kg/h); L, landings $(\mathrm{kg} / \mathrm{h}) . \mathrm{S}, J^{\prime}$ and $H^{\prime}$ are calculated considering only the fish species. $\mathrm{D}$ and $\mathrm{L}$ are calculated from the total catch.

\begin{tabular}{lcccccc}
\hline & $\begin{array}{c}\text { SS } \\
(70-88 \mathrm{~m})\end{array}$ & $\begin{array}{c}\mathrm{DS} \\
(155-180 \mathrm{~m})\end{array}$ & $\begin{array}{c}\mathrm{SB} \\
(196-276 \mathrm{~m})\end{array}$ & $\begin{array}{c}\text { US } \\
(307-410 \mathrm{~m})\end{array}$ & $\mathrm{p}$ & Post-hoc \\
\hline $\mathrm{n}$ & 12 & 4 & 8 & 4 & & \\
$\mathrm{~s}$ & 55 & 46 & 51 & 33 & & $\mathrm{p}=\mathrm{DS}=\mathrm{SB}=\mathrm{US}$ \\
$\mathrm{S}$ & $26.00 \pm 3.33$ & $28.25 \pm 3.59$ & $23.75 \pm 3.81$ & $24.50 \pm 3.70$ & $\mathrm{p}>0.05$ & $\mathrm{SS}=\mathrm{US}>\mathrm{SB}=\mathrm{DS}$ \\
$J^{\prime}$ & $0.63 \pm 0.09$ & $0.47 \pm 0.04$ & $0.46 \pm 0.09$ & $0.68 \pm 0.01$ & $\mathrm{p}<0.0001$ & $\mathrm{SS}=\mathrm{US}>\mathrm{SB}=\mathrm{DS}$ \\
$H^{\prime}$ & $2.04 \pm 0.27$ & $1.58 \pm 0.18$ & $1.46 \pm 0.28$ & $2.18 \pm 0.14$ & $\mathrm{p}<0.0001$ & $\mathrm{SS}>\mathrm{DS}=\mathrm{SB}=\mathrm{US}$ \\
$\mathrm{D}$ & $32.89 \pm 13.13$ & $14.03 \pm 2.8$ & $12.72 \pm 13.56$ & $10.77 \pm 1.84$ & $\mathrm{p}<0.01$ & $\mathrm{SS}=\mathrm{DS}=\mathrm{SB}=\mathrm{US}$ \\
$\mathrm{L}$ & $45.64 \pm 19.3$ & $31.04 \pm 3.8$ & $25.37 \pm 9.19$ & $35.37 \pm 20.01$ & $\mathrm{p}>0.05$ & \\
\hline
\end{tabular}
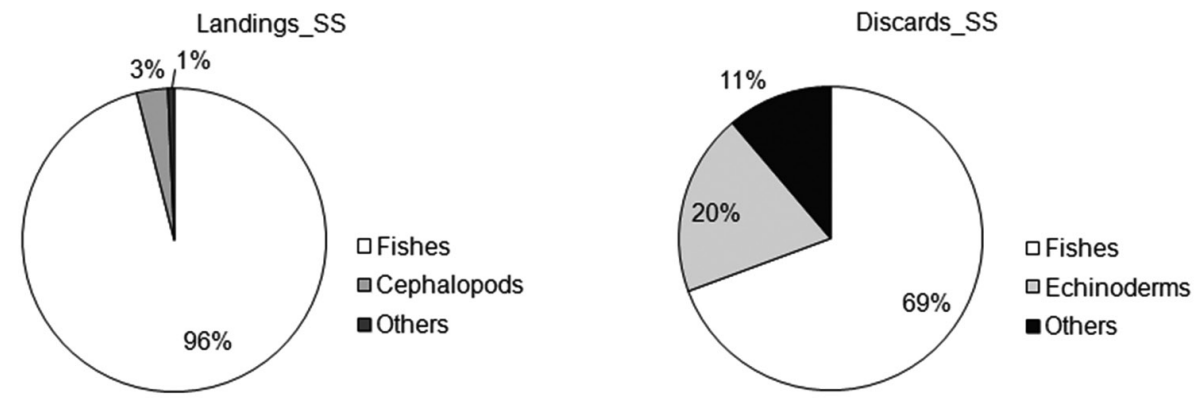

Landings_DS

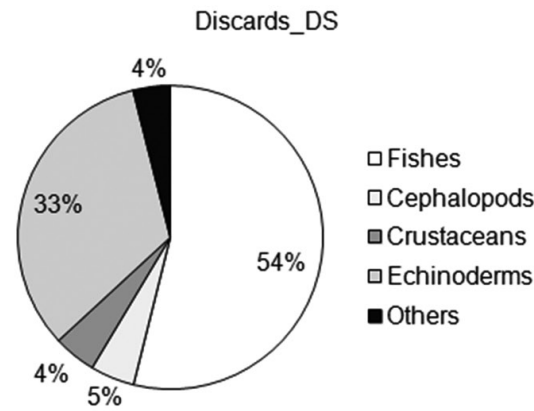

Landings_SB

Discards_SB

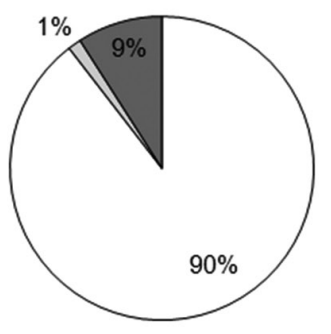

口Fishes

$\square$ Cephalopods

口Crustaceans

Landings_US
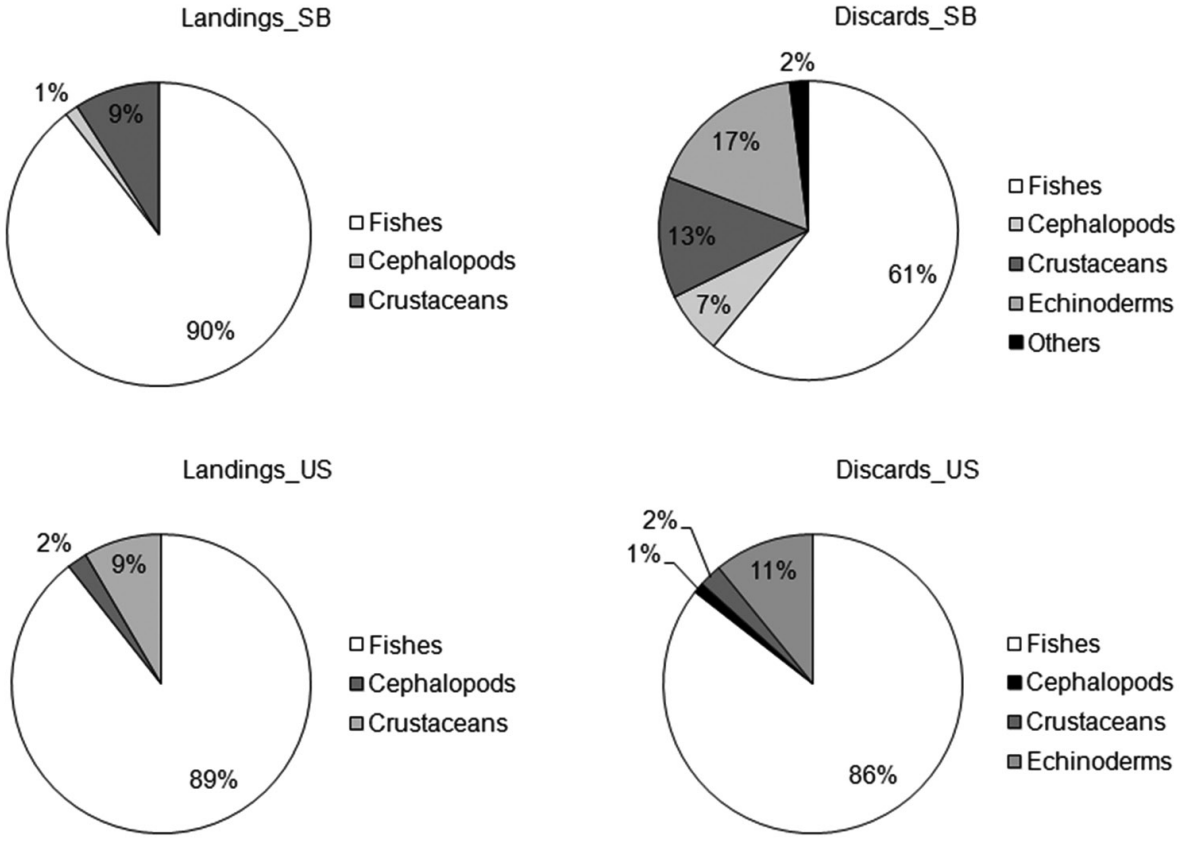

Fig. 3. - Biomass composition for landed and discarded groups of trawl samples obtained from cluster analyses. SS, shallow shelf; DS, deep shelf; SB, shelf break; US, upper slope. 
Table 4. Abundance (N, individuals/h) and biomass (B, $\mathrm{kg} / \mathrm{h}$ ) of landings and discards of fishes and invertebrates in the four groups identified in the cluster analysis. SS, shallow shelf; DS, deep shelf; SB, shelf break; US, upper slope. *discarded fraction of marketable species. \% D, percentage represent by discards in relation to the total catch.

\begin{tabular}{|c|c|c|c|c|c|c|c|c|c|c|c|}
\hline & \multicolumn{4}{|c|}{ Landings } & \multicolumn{7}{|c|}{ Discards } \\
\hline SS (70-88 m) & & & & & & SS (70-88 m) & & & & & \\
\hline Fishes & $\stackrel{\mathrm{N}}{\mathrm{N}}$ & $\pm \mathrm{SD}$ & B & $\pm{ }_{5} \mathrm{SD}$ & B\% & Fishes & $\stackrel{\mathrm{N}}{\mathrm{N}}$ & $\pm \mathrm{SD}$ & B & $\pm \mathrm{SD}$ & B\% \\
\hline Mullus barbatus & 195.48 & 136.63 & 9.99 & 5.58 & 21.89 & Pagellus bogaraveo* & 275.46 & 340.93 & 7.32 & 9.31 & 22.25 \\
\hline Dentex maroccanus & 89.76 & 114.83 & 6.22 & 8.29 & 13.63 & Dipturus oxyrinchus & 0.63 & 0.73 & 3.52 & 5.39 & 10.71 \\
\hline Pagellus acarne & 114.69 & 169.57 & 6.07 & 8.8 & 13.29 & Lepidotrigla cavillone & 153.83 & 162.17 & 2.08 & 2.08 & 6.32 \\
\hline Merluccius merluccius & 35.49 & 34.95 & 5.25 & 4.98 & 11.51 & Scyliorhinus canicula & 7.3 & 6.10 & 2.06 & 1.27 & 6.25 \\
\hline Pagellus erytrinus & 73.05 & 31.42 & 4.98 & 2.34 & 10.91 & Rostraraja alba & 0.45 & 1.11 & 1.3 & 4.20 & 3.95 \\
\hline Lophius budegassa & 2.13 & 1.59 & 2.52 & 2.52 & 5.52 & Scyliorhinus stellaris & 0.86 & 2.30 & 0.9 & 1.73 & 2.74 \\
\hline Boops boops & 44.74 & 27.85 & 2.45 & 1.43 & 5.37 & Citharus linguatula & 45.7 & 54.42 & 0.83 & 1.07 & 2.51 \\
\hline Raja clavata & 0.89 & 0.91 & 1.45 & 1.41 & 3.18 & Spicara smaris & 44.77 & 45.04 & 0.72 & 0.73 & 2.2 \\
\hline Zeus faber & 0.9 & 1.28 & 0.96 & 1.34 & 2.1 & Raja miraletus & 1.53 & 2.34 & 0.64 & 0.96 & 1.94 \\
\hline Dentex dentex & 0.26 & 0.69 & 0.91 & 2.25 & 1.99 & Dasyatis pastinaca & 0.33 & 0.89 & 0.55 & 1.84 & 1.66 \\
\hline Pagellus bogaraveo & 18.35 & 43.51 & 0.54 & 1.31 & 1.19 & Pagellus erytrinus & 11.02 & 12.16 & 0.33 & 0.36 & 1.02 \\
\hline Mullus surmuletus & 7.35 & 17.25 & 0.51 & 1.07 & 1.12 & Others & 68.66 & 88.17 & 2.69 & 4.67 & 8.18 \\
\hline Chelidonichthys lucernus & 1.16 & 1.37 & 0.46 & 0.67 & 1.01 & Invertebrates & & & & & \\
\hline Others & 26.32 & 54.4 & 1.51 & 3.12 & 3.32 & Stichopus regalis & 40.68 & 47.15 & 6.41 & 6.40 & 19.49 \\
\hline Invertebrates & & & & & & Echinoderms & 22.51 & 24.71 & 2.06 & 4.52 & 6.26 \\
\hline Cephalopods & 8.87 & 4.6 & 1.51 & 0.71 & 3.31 & Tunnicate & 5.79 & 5.08 & 0.52 & 0.55 & 1.57 \\
\hline \multirow[t]{2}{*}{ Others } & 0.44 & 1.1 & 0.31 & 1.07 & 0.68 & Cephalopods & 10.67 & 10.27 & 0.42 & 0.39 & 1.27 \\
\hline & & & & & & $\begin{array}{l}\text { Porifera } \\
\% \mathrm{D}=42.02\end{array}$ & & & 0.41 & 0.46 & 1.25 \\
\hline DS $(155-180 \mathrm{~m})$ & & & & & & DS (155-180 m) & & & & & \\
\hline Fishes & $\mathrm{N}$ & $\pm \mathrm{SD}$ & B & $\pm \mathrm{SD}$ & $\mathrm{B} \%$ & Fishes & $\mathrm{N}$ & $\pm \mathrm{SD}$ & B & $\pm \mathrm{SD}$ & $\mathrm{B} \%$ \\
\hline Merluccius merluccius & 228.92 & 49.4 & 17.63 & 1.78 & 56.78 & Merluccius merluccius* & 100.11 & 80.28 & 1.96 & 1.77 & 13.99 \\
\hline Pagellus bogaraveo & 151.33 & 123.01 & 4.74 & 3.78 & 15.27 & Pagellus bogaraveo** & 38.76 & 36.57 & 1.07 & 1.03 & 7.64 \\
\hline Trachurus trachurus & 66.97 & 111.12 & 1.31 & 0.97 & 4.22 & lavata & 4.1 & 1.2 & 0.95 & 1.21 & 6.79 \\
\hline Mullus barbatus & 16.22 & 12.43 & 1.04 & 0.97 & 3.37 & Citharus linguatula* & 64.39 & 58.75 & 0.89 & 0.77 & 6.38 \\
\hline Lophius budegassa & 1.94 & 1.51 & 0.74 & 0.26 & 2.39 & Dasyatis pastinaca & 0.09 & 0.17 & 0.69 & 1.38 & 4.92 \\
\hline Trigla lyra & 4.2 & 2.5 & 0.53 & 0.33 & 1.7 & Raj & 0.21 & 0.42 & 0.6 & 1.21 & 4.31 \\
\hline Micromesistius poutassou & 12.98 & 18.77 & 0.52 & 0.75 & 1.67 & stellaris & 0.19 & 0.22 & 0.22 & 0.41 & $\begin{array}{l}4.31 \\
1.58\end{array}$ \\
\hline Others & 3.62 & 4.04 & 0.67 & 0.64 & 2.17 & Scyliorhinus canicula & 1.54 & 0.78 & 0.21 & 0.18 & 1.51 \\
\hline Invertebrates & & & & & & Squalus acanthias & 0.1 & 0.21 & 0.16 & 0.31 & 1.11 \\
\hline Cephalopods & 12.44 & 20.33 & 0.81 & 1.13 & 2.62 & Others & 48.59 & 47.06 & 0.69 & 0.7 & 4.9 \\
\hline Parapenaeus longirostris & 589.16 & 362.21 & 3.02 & 1.86 & 9.74 & Invertebrates & & & & & \\
\hline \multirow[t]{7}{*}{ Nephrops norvegius } & 0.1 & 0.21 & 0.02 & 0.04 & 0.07 & Stichopus regalis & 12.83 & 7.52 & 4.55 & 3.09 & 32.43 \\
\hline & & & & & & Cephalopods & 26.54 & 25.7 & 0.65 & 0.49 & 4.62 \\
\hline & & & & & & Porifera & & & 0.55 & 1.09 & 3.9 \\
\hline & & & & & & Parapenaeus longirostris* & 104.96 & 122.03 & 0.43 & 0.5 & 3.08 \\
\hline & & & & & & Other Echinoderms & 2.17 & 4.07 & 0.2 & 0.31 & 1.45 \\
\hline & & & & & & Others & 6.02 & 5.93 & 0.2 & 0.33 & 1.39 \\
\hline & & & & & & $\mathrm{D} \%=30.81$ & & & & & \\
\hline SB (196-276 m) & & & & & & SB (196-276 m) & & & & & \\
\hline Fishes & $\mathrm{N}$ & $\pm \mathrm{SD}$ & B & $\pm \mathrm{SD}$ & $\mathrm{B} \%$ & & $\mathrm{~N}$ & $\pm \mathrm{SD}$ & B & $\pm \mathrm{SD}$ & B\% \\
\hline Merluccius merluccius & 165.44 & 67.92 & 19.79 & $\overline{7} .38$ & 77.98 & Scyliorhinus & 23.5 & 21.68 & 2.64 & 2.42 & 20.77 \\
\hline Pagellus bogaraveo & 24.63 & 59.1 & 0.97 & 2.37 & 3.81 & $M u s$ & 0.44 & 1.12 & 1.85 & 4.93 & 14.56 \\
\hline Loph & 1.2 & 0.59 & 0.61 & 0.26 & 2.39 & & 0.42 & 0.48 & 0.51 & 0.6 & 4.05 \\
\hline Phycis blennoides & 4.69 & 3.97 & 0.42 & 0.35 & 1.64 & merluccius* & 32.1 & 33.94 & 0.5 & 0.5 & 3.9 \\
\hline Others & 8.2 & 10.69 & 0.94 & 1.35 & 3.7 & Raja clav & 0.52 & 0.96 & 0.34 & 0.75 & 2.68 \\
\hline rates & & & & & & Pagellus bogaraveo* & 8.39 & 11.65 & 0.3 & 0.51 & 2.39 \\
\hline Cephalopods & 4.33 & 4.31 & 0.36 & 0.23 & 1.41 & Myl & 0.02 & 0.07 & 0.24 & 0.68 & 1.89 \\
\hline Parapenaeus longirostris & 443.2 & 335.2 & 2.3 & 1.75 & 9.06 & Torpedo marmorata & 0.33 & 0.53 & 0.15 & 0.32 & 1.15 \\
\hline & & & & & & Others & 60.07 & 75.47 & 1.15 & 1.89 & 9.07 \\
\hline & & & & & & $\begin{array}{l}\text { Invertebrates } \\
\text { Stichopus regalis }\end{array}$ & 11.41 & 14.66 & 2.17 & 3.11 & 17.04 \\
\hline & & & & & & Parapenaeus longirostris* & $\begin{array}{l}1.41 \\
488.52\end{array}$ & 720.45 & 1.44 & & 11.33 \\
\hline & & & & & & Cephalopods & 25. & 21.27 & 0.85 & 1.02 & 6.69 \\
\hline & & & & & & Pol & & & 0.18 & 0.42 & 1.44 \\
\hline & & & & & & Others & 51.86 & 48.3 & 0.39 & 0.53 & 3.05 \\
\hline & & & & & & $\mathrm{D} \%$ & & & & & \\
\hline US (307-410 m) & & & & & & US (307-410 m) & & & & & \\
\hline Fishes & $\mathrm{N}$ & $\pm \mathrm{SD}$ & B & $\pm \mathrm{SD}$ & $\mathrm{B} \%$ & Fis & $\mathrm{N}$ & $\pm \mathrm{SD}$ & B & $\pm \mathrm{SD}$ & B\% \\
\hline & 49.95 & 38.41 & 13.14 & 4.01 & 37.14 & Sc & 15.14 & 6.74 & 3.12 & 1.07 & 29 \\
\hline nesistius poutassou & 85.31 & 92.82 & 12.06 & 14.78 & 34.1 & lorinchus & 148.73 & 49.91 & 1.49 & 0.53 & 13.87 \\
\hline & 22.16 & 29.9 & 2.16 & 2.26 & 6.1 & $\mathrm{Hol}$ & 56.06 & 10.11 & 0.93 & 0.69 & 8.6 \\
\hline blenn & 12.4 & 7.43 & 1.81 & 1.85 & 5.13 & Ga & 23 & 16.74 & 0.72 & 0.53 & 6.65 \\
\hline & 1.55 & 1.27 & 0.83 & 0.71 & 2.35 & & 20.73 & 5.44 & 0.5 & 0.13 & 4.64 \\
\hline Lepidorhomus boscii & 2.94 & 1.46 & 0.51 & 0.33 & 1.43 & Squ & 0.38 & 0.51 & 0.41 & 0.66 & 3.82 \\
\hline Helicolenus dactylepterus & 3.69 & 3.59 & 0.48 & 0.48 & 1.36 & oxyrinchus & 0.38 & 0.3 & 0.39 & 0.37 & 3.6 \\
\hline Other & 2.26 & 2.19 & 0.63 & 0.91 & 1.78 & Molva dypterygia* & & 4.41 & 0.33 & 0.14 & 3.1 \\
\hline & & & & & & Etmopterus spinax & 5.97 & 1.74 & 0.22 & 0.06 & 2.09 \\
\hline Cephalopods & 4.35 & 4.16 & 0.77 & 0.9 & 2.17 & 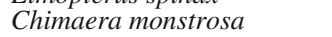 & 1.6 & 1.11 & 0.21 & 0.31 & 1.95 \\
\hline eus longirostris & 506.69 & 96.36 & 2.83 & 0.67 & 8 & Lepidorhombus boscii* & 8.97 & 5.51 & 0.16 & 0.11 & 1.44 \\
\hline Nephrops norvegius & 2.47 & 2.33 & 0.15 & 0.11 & 0.43 & Others & 58.11 & 32.09 & 0.73 & 0.57 & 6.77 \\
\hline & & & & & & Invertebrates & & & & & \\
\hline & & & & & & Stichopus regalis & 5.44 & 4.25 & 1.17 & 1.02 & 10.86 \\
\hline & & & & & & enaeus longirostris* & 51.96 & 103.93 & & & 2.4 \\
\hline & & & & & & Cephalopods & 5.18 & & 0.12 & 0.24 & 1.13 \\
\hline & & & & & & $\begin{array}{l}\text { Others } \\
D \%=23.24\end{array}$ & 1.16 & 2.32 & 0.01 & 0.02 & 0.07 \\
\hline
\end{tabular}



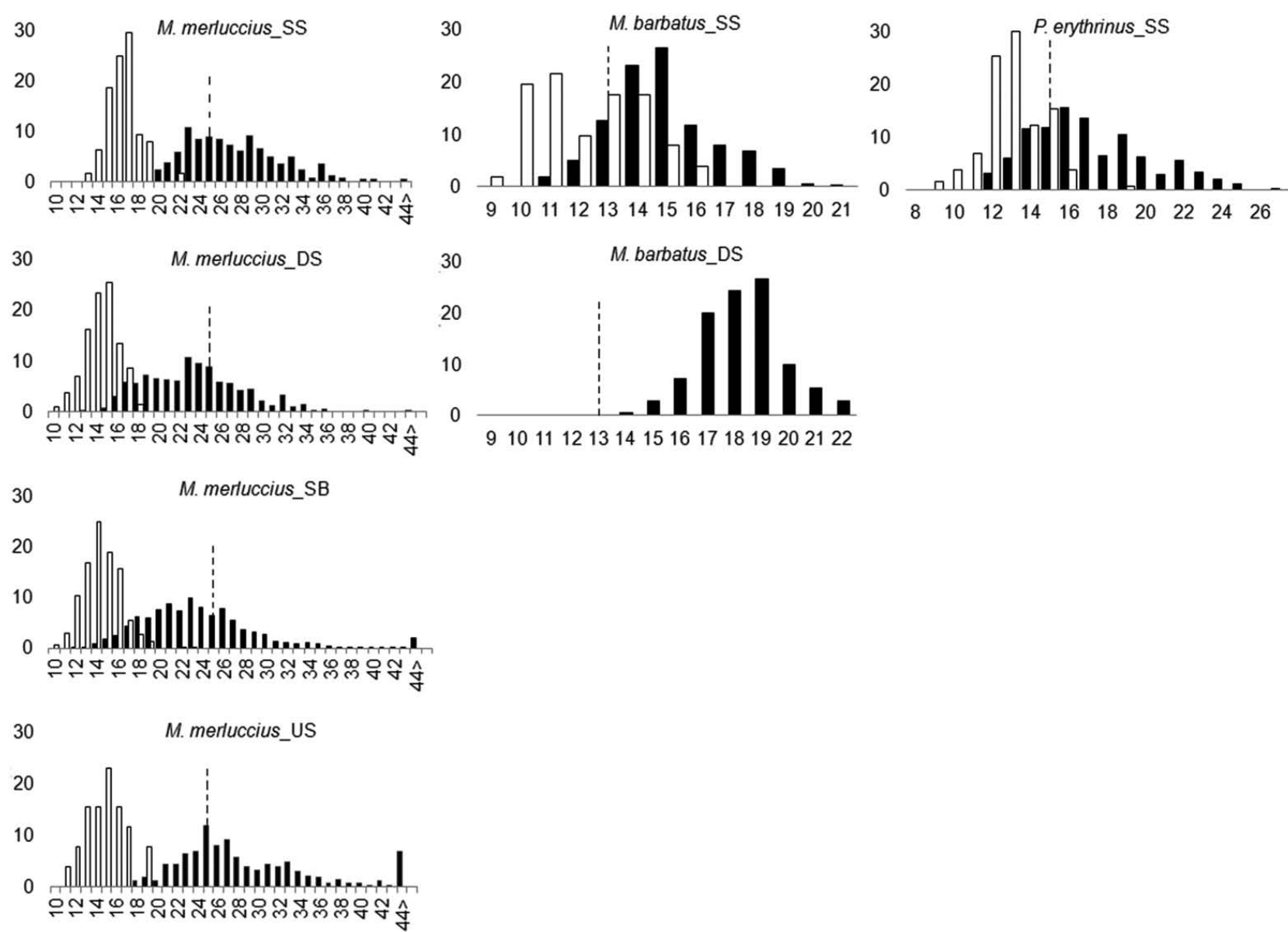

Fig. 4. - Size composition of M. merluccius, M. barbatus and P. erythrinus by depth strata (SS, shallow shelf; DS, deep shelf; SB, shelf break; US, upper slope), showing the landed (black bars) and discarded (white bars) fractions. The discontinuous line shows the current minimum legal landing size.

Similarly, fishes accounted for $90 \%$ of landings from both the SB and the US, M. merluccius (78\%), and M. merluccius and Micromesistius poutassou (Risso) (both summing $71 \%$ ) being the most important species, respectively. The total amount of invertebrates decreased with depth, with maximum values in the SS. $P$. longirostris was the main species landed on the DS, SB and US, representing $8 \%$ to $10 \%$ of the landings.

The fish biomass discarded ranged between $53 \%$ on the DS and $86 \%$ on the US (Table 4). P. bogaraveo, Dipturus oxyrinchus (L.), L. cavillone and S. canicula were the dominant fish, accounting for $46 \%$ of discards on the SS (Table 4). On the DS, M. merluccius, P. bogaraveo, Raja clavata L. and C. linguatula accounted for $35 \%$ of discards, while on the SB S. canicula and Mustelus mustelus (L.) were the main species (35\%). $S$. canicula, $C$. caelorinchus, $H$. mediterraneus and Galeus melastomus Rafinesque 1810 accounted for $58 \%$ of discards on the US (Table 4). Within invertebrates, echinoderms were the main group in discards along the whole depth range surveyed, with Stichopus regalis (Cuvier) as the main species, ranging from $11 \%$ on the US to $34 \%$ on the DS (Table 4). P. longirostris also accounted for a large proportion of the discards on the SB $(11 \%)$.

Size of landed and discarded specimens of M. merluccius ranged from 12 to $99 \mathrm{~cm}$ (mode: $23 \mathrm{~cm}$ ) and from 10 to $23 \mathrm{~cm}$ (mode: $14 \mathrm{~cm}$ ), respectively. More than half of $M$. merluccius landed individuals (55\%) were below the minimum legal size (MLS=25 cm; Fig. 4). Considering all bathymetric strata, this was the only species for which all specimens landed were of marketable size. Landed size of $M$. barbatus ranged from 11 to $22 \mathrm{~cm}$ (mode: $15 \mathrm{~cm}$ ), while discarded size ranged from 9 to $16 \mathrm{~cm}$ (mode: $11 \mathrm{~cm}$ ). On the DS, landed size of this species was clearly higher than its MLS $(13 \mathrm{~cm})$, and only $6 \%$ of $M$. barbatus landed from the SS was lower than the MLS, while $10 \%$ of discarded specimens were higher than the MLS. P. erythrinus was only caught from the SS and the specimens were mostly $(77 \%)$ larger than the MLS $(15 \mathrm{~cm})$. Landed and discarded size ranged from 12 to $27 \mathrm{~cm}$ (mode: 16 $\mathrm{cm}$ ) and from 9 to $19 \mathrm{~cm}$ (mode: $13 \mathrm{~cm}$ ), respectively.

Landings size of $D$. maroccanus specimens ranged from 12 to $22 \mathrm{~cm}$ (mode: $18 \mathrm{~cm}$ ), while discards ranged from 6 to $16 \mathrm{~cm}$ (mode: $13 \mathrm{~cm}$ ). Ranges of landed and discarded specimens of $P$. acarne were $13-22 \mathrm{~cm}$ (mode: $16 \mathrm{~cm}$ ) and 13-19 cm (mode: $15 \mathrm{~cm}$ ), respectively; those of $P$. bogaraveo were 11-25 cm (mode: 13 $\mathrm{cm}$ ) and 10-18 cm (mode: $13 \mathrm{~cm}$ ), respectively; those of $M$. poutassou were $15-31 \mathrm{~cm}$ (mode: $19 \mathrm{~cm}$ ) and 15$21 \mathrm{~cm}$ (mode: $18 \mathrm{~cm}$ ), respectively; those of Lophius budegassa Spinola were 13-52 cm (mode: $24 \mathrm{~cm}$ ) and 9-27 cm (mode: $14 \mathrm{~cm}$ ), respectively; and those of $P$. 

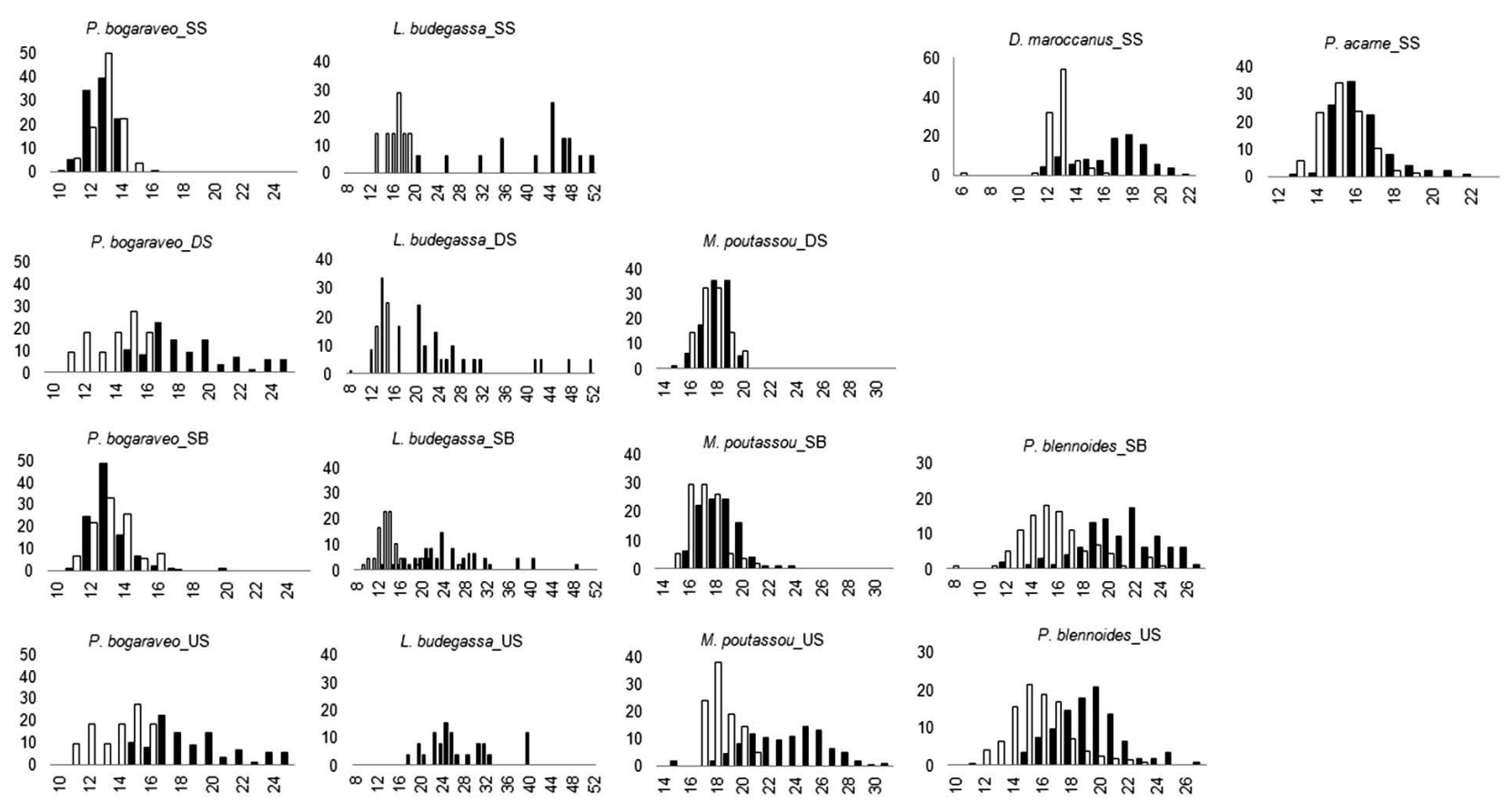

Fig. 5. - Size composition of main species (P. bogaraveo, L. budegassa, D. maroccanus, P. acarne, M. poutassou and P. blennoides) without minimum legal landing size by depth strata (SS, shallow shelf; DS, deep shelf; SB, shelf break; US, upper slope), showing the landed (black bars) and discarded (white bars) fractions.

blennoides were $12-52 \mathrm{~cm}$ (mode: $20 \mathrm{~cm}$ ) and $8-24 \mathrm{~cm}$ (mode: $15 \mathrm{~cm}$ ), respectively (Fig. 5).

\section{DISCUSSION}

Depth zonation has been described as a main factor affecting faunal change on the continental margins (see Carney 2005 for a general review). In the Mediterranean, numerous macrofaunal zonation studies have been developed in the past three decades (e.g. Cartes and Sardà 1993, Stefanescu et al. 1993, Massutí et al. 1996, D’Onghia et al. 1998, Moranta et al. 1998, Kallianiotis et al. 2000, Labrapoulou and Papacontantinou 2000). In this study, although the survey was performed in a commercial fishing vessel under commercial fishing conditions, four fish assemblages associated with different bathymetric strata have been detected in the fishing grounds around Gökçeada Island in the northeastern Aegean Sea, similar to those found in other areas of the Mediterranean Sea (e.g. Kallianiotis et al. 2004, Labropoulou and Papaconstantinou 2004, Massutí and Reñones 2005, Moranta et al. 2008).

The species compositions on the SS and the DS around Gökçeada Island are in accordance with the results obtained in terms of dominant species in the southern North Aegean Trough, with M. barbatus on the SS and M. merluccius on the DS between the most abundant ones (Labrapoulou and Papaconstantinou 2000, 2004, Kallianiotis et al. 2004, Keskin et al. 2011a). Nevertheless, subdominant species changed from one area to another and even between adjacent bottoms of the mainland coast. The main difference on the continental shelf is related to the family Sparidae, with a high number of species and high abundance on the SS. In our study area, five of the ten most important species in abundance belonged to this family $[P$. bogaraveo, $P$. acarne, $D$. maroccanus, P. erythrinus and Boops boops (L.)]. By contrast, in the surrounding areas these species are not among the most abundant, whereas other species such as Serranus hepatus (L.) and T. minutus are more abundant (Labrapoulou and Papaconstantinou 2004, Keskin et al. 2011a). These differences could be related to the bottom morphology of Gökçeada Island, with a large proportion of rocky areas nearby to fishing grounds and lower presence of muddy bottoms on the SS (Bayhan et al. 2001, Koral et al. 2008). Other environmental factors such as high productivity, river runoff, salinity and temperature along the Aegean Sea have also been suggested as important descriptors for fish community structure of the area (Stergiou and Polard 1994, Kallianiotis et al. 2004).

Fish assemblages found on the SB and the US are characterized by the high abundance of both $M$. merluccius and $P$. bogaraveo. The abundance of M. merluccius around Gökçeada Island, and in Saros Bay (Keskin et al. 2011a), is higher than in other areas of the Aegean Sea (Labropoulou and Constantinou 2004, 2005). This could be related to different environmental factors. It is important to highlight the possible effect of the mixture of Levantine Intermediate Water and Black Sea Water between 20 and $100 \mathrm{~m}$ depth in the northeastern Aegean Sea, where high nutrient concentration and production in the upper layers has been described (Pazi 2008). This high productivity can increase the abundances of small pelagics, which have been identified as the main trophic source of M. merluccius in this area (mainly sardine and anchovy, Tsagarakis et al. 2010, Harmelin-Vivien et al. 2012). Moreover, the spill over from the nearby protected area in Saros Bay, where bottom trawling has been banned since 1993 , could also increase the abundance of M. merluccius. 
By contrast, the higher abundance of $P$. bogaraveo could be related to the particular characteristics of the sea bottom in the study area mentioned above.

For the unexploited demersal stocks in Saros Bay, İşmen et al. (2010) reported total catch of $111.4 \mathrm{~kg} / \mathrm{h}$ from 50 to $100 \mathrm{~m}$ depth, $436.8 \mathrm{~kg} / \mathrm{h}$ from 100 to 200 $\mathrm{m}$ and $565.5 \mathrm{~kg} / \mathrm{h}$ from 200 to $500 \mathrm{~m}$. These yields, obtained during the same season as the present study (winter), are clearly higher than our results, which range from $78.5 \mathrm{~kg} / \mathrm{h}$ in the SS to $46.1 \mathrm{~kg} / \mathrm{h}$ in the US, suggesting that fishing impact is the main causative factor of these differences. Nevertheless, other environmental factors operating at a regional scale could also affect these differences, as mentioned above.

The higher values obtained for the diversity indexes $H^{\prime}$ and $J$ ' on the SS and the US could be related to the high productivity and the bottom characteristics of the shallow continental shelf mentioned above and to the fishing strategy followed by the bottom trawl fleet in the area, which exerts a higher fishing effort on the DS and the SB than on the US.

Bottom trawling has a large impact on Mediterranean ecosystems, because of the low selectivity of the fishing gears used and the high generation of discards they can produce, which result in the mortality of small size specimens of marketable species and unmarketable species living on the bottom (Stergiou et al. 1998, Moranta et al. 2000, Tudela 2004). Tsagarakis et al. (2013) estimated that the amount of discards was around $230000 \mathrm{t}$ (18.6\% of catch) in the Mediterranean Sea. According to these authors, discarding varies highly along the basin both geographically and among fishing gears, with bottom trawls being responsible for the bulk of discards, since they are characterized by high discard ratios. In addition, discarding in the Mediterranean is regulated by market demands and to a lesser extent by legal constraints (see comments below on MLS), and marketable by-catch may be an important supplemental source of income (Tsagarakis et al. 2013). Therefore, the results obtained in this study with regard to the percentage of discards and the rate of discarded species may be related to fishers' strategy to respond to these market demands. Because of their higher prices in the market some species, such as $M$. merluccius, can be classified as target species for the bottom trawl fisheries. By contrast, other species have a lower demand (e.g. Pagellus acarne) or even no demand (e.g. S. canicula), and are always discarded.
Various studies on trawl fishery showed that discard rates in some regions of the Mediterranean Sea ranged between $20 \%$ and $70 \%$ of the total catch, depending on depth and the associated macro-epibenthic communities, with higher rates in shallow fishing grounds than in deeper ones (Table 5). For example, deep sea shrimp trawl fisheries generate lesser discards than shallow water fisheries targeting stripped red mullets Mullus surmuletus and red mullets M. barbatus in the Balearic Islands and the Catalan coast, as well as intermediate water fisheries targeting hake Merluccius merluccius (Tsagarakis et al. 2013). The percentage of discards from the total catch at Gökçeada Island ranged between $23 \%$ and $42 \%$, with a decreasing trend with depth and fishes and echinoderms (mainly $S$. regalis) as the two main groups in all bathymetric strata, also on the SS. The amount of crustaceans, mainly $P$. longirostris, in the discards as well as in the landings is not very great. Yields obtained in the present study for $P$. longirostris are much lower $(\sim 3 \mathrm{~kg} / \mathrm{h})$ than those obtained for the unexploited stock of this species in Saros Bay (>50 kg/h; Bilgin et al. 2009). Kara and Gurbet (1999) identified $P$. longirostris as the main exploited species from the SB and the US in the study area, with yields of $17 \mathrm{~kg} / \mathrm{h}$. Taking into account that similar fishing gears with a codend mesh size of $22 \mathrm{~mm}$ were used in these studies, the observed differences in the yields cannot be attributed to different catchability of the gears. Fishing pressure appears the most likely factor affecting abundances of $P$. longirostris in the area.

The management of bottom trawl fishery in Turkish waters is mainly based on technical measures, such as minimum landing sizes for some species, minimum mesh size $(40 \mathrm{~mm}$ in the Black Sea and $44 \mathrm{~mm}$ in the Aegean Sea, both diamond mesh type), and closed areas and seasons (Anonymous 2012). MLS is also applied to protect recruitment and juveniles of exploited species and is usually below the first maturity size (FMS). According to current regulation, the MLS for M. merluccius, M. barbatus and $P$. erythrinus is 25 , 13 and $15 \mathrm{~cm}$, respectively (Anonymous 2012), while the FMS of these species in the northeastern Aegean Sea ranges between 27 and $34 \mathrm{~cm}$ for $M$. merluccius, and is around $12 \mathrm{~cm}$ for $M$. barbatus and $14 \mathrm{~cm}$ for $P$. erythrinus (İsmen et al. 2010). Although the MLS seems to be correct for M. barbatus and P. erythrinus, most of the specimens of $M$. merluccius caught in the present study were below its FMS, so the current MLS

Table 5. - Summary of bibliographic information on the percentages of discarded catch produced by bottom trawl fisheries by depth stratum in different areas of the Mediterranean. S, square mesh; DI, diamond mesh; W MED, western Mediterranean; E MED, eastern Mediterranean.

\begin{tabular}{|c|c|c|c|c|c|c|c|}
\hline \multirow{2}{*}{ Region } & \multirow{2}{*}{ Reference } & \multirow{2}{*}{ Year } & \multirow{2}{*}{ Mesh size } & \multicolumn{4}{|c|}{$\%$ discard of total catch } \\
\hline & & & & $<150 \mathrm{~m}$ & $151-350 \mathrm{~m}$ & $>350 \mathrm{~m}$ & Av. \\
\hline \multirow[t]{7}{*}{ W MED } & Carbonell et al. (1998) & $1995-96$ & 40DI & $23-67$ & $13-62$ & $14-43$ & \multirow{7}{*}{42} \\
\hline & Moranta et al. (2000) & $1996-97$ & 40DI & & & $38-58$ & \\
\hline & D’Onghia et al. (2003) & $1996-98$ & 40DI & & & $23-45$ & \\
\hline & Sánchez et al. (2004) & $1995-96$ & 40DI & 62.7 & 19.35 & 19.5 & \\
\hline & Sánchez et al. (2007) & 2002-04 & 40DI & $39-46$ & & & \\
\hline & Guijarro and Massutí (2006) & $2002-03$ & 40(DI/S) & & & $18-45 / 9-10$ & \\
\hline & Ordines et al. (2006) & 2002-03 & $40(\mathrm{DI} / \mathrm{S})$ & $30 / 70$ & & & \\
\hline \multirow[t]{5}{*}{ E MED } & Stergiou et al. (1998) & 1993-94 & $28 \mathrm{DI}$ & & & & \multirow[t]{2}{*}{45} \\
\hline & Vassilopoulou and Papaconstantinou (1998) & 1995 & 28DI & $53-59$ & $41-63$ & 37 & \\
\hline & Machias et al. (2001) & $1995-98$ & 28DI & & & & \multirow[t]{2}{*}{44} \\
\hline & Duruer and Tosunoğlu (2012) & 2008 & 44DI/40S & $30 / 20$ & & & \\
\hline & This study & $2009-10$ & 44DI & 42 & $31-33$ & 23 & 37 \\
\hline
\end{tabular}


is not adequate to ensure that juveniles of this species reach maturity and thus contribute to the spawning stock. Moreover, one of the most abundant fish species in all depth strata, $P$. bogaraveo, has no size regulation in Turkish waters. This species is clearly caught below the FMS in both sexes, 26-29 in males and 28-32 cm in females (Estácio et al. 2001, Krug 1998). For the Mediterranean little information about the FMS of $P$. bogaraveo is available, but in the Atlantic the FMS has been estimated to stand at approximately $24 \mathrm{~cm}$ (Chilari et al. 2006). Assuming the same FMS for the Mediterranean, almost all the individuals caught during the present study would be below the FMS. Hence, high mortality rates of young immature individuals of this commercially important species (Chilari et al. 2006) have to be discarded or sold at low prices.

The danger of increasing discarding through a heavy focus on regulating the MLS has been recognized (Caddy, 2009). Moreover, several bottom trawl selectivity studies have demonstrated that high discard rates, with a large proportion of immature small size specimens and non-commercial invertebrates, is a general problem of the trawl fishery in the Mediterranean Sea (e.g. Stergiou et al. 1997, 1998, Vassiloupou and Papaconstantinou 1998, Sánchez et al. 2004, Guijarro and Massutí 2006, Ordines et al. 2006). These studies showed that replacing the $40-\mathrm{mm}$ diamond mesh with the 40-mm square mesh in the codend improves the selectivity of bottom trawling while maintaining commercial yields for most of the species. In fact, this change of mesh is a mandatory regulation for the Mediterranean that has been enforced since 2008 for the EU member states (Council Regulation EC n 1967/2006). In addition, the General Fisheries Commission for the Mediterranean recommended that the Members and cooperating entities should adopt and implement a minimum $40-\mathrm{mm}$ square mesh codend or a diamond mesh size of at least $50 \mathrm{~mm}$ for all trawling activities (REC.CM-GFCM/33/2009/2). Though the minimum mesh size for trawl nets in Turkey is larger than in other areas of the Mediterranean (44 mm), the high amount of discards estimated in the present study indicates that it is not sufficient to reduce the discards significantly. Changing mesh geometry in the codend, from diamond to square, as in other areas of the Mediterranean, could improve selectivity in the Turkish bottom trawl fishery. However, the consequences of this change should previously be evaluated in order to take into account the particularities of the area.

Finally, it should be noted that the results of the present study provide only some preliminary indications and that other scientific surveys based on longer time series in the area are needed to support these findings. The issue of discards cannot be handled only with selective fishing, since this can affect food web structure and ecosystem functioning (Tsagarakis et al. 2013). Thus, mitigation of discards should be based on a combination of measures and, although the results of this study are purely indicative, the fact that a large proportion of juveniles of target species (e.g. Merluccius merluccius) are caught suggests that other initiatives should be implemented, such as temporal restrictions on fishing activities or the establishment of closed areas of ecological importance for the maintenance of heavily exploited populations and/or species of conservation concern.

\section{ACKNOWLEDGEMENTS}

This work was supported by the Scientific Research Projects Coordination Unit of Istanbul University (Project number: 4365; Project number: 6121; Project number: 26838). The authors thank PhD students Emre Yemişgen and Onur Gönülal (Institute of Science, University of Istanbul) and colleagues of the Centre Oceanogràfic de les Baleares (Instituto Español de Oceanografía) for their help during the sampling and the writing of the manuscript. The authors also thank Prof. Bayram Öztürk (Head of Department of Marine Biology, Faculty of Fisheries, University of Istanbul) for his support during the study and Hilmar Hinz for revising the last version of the manuscript.

\section{REFERENCES}

Anonymous 2012. Commercial fish catching regulation in seas and inland waters in 2012-2016 fishing period: Circular No 2012/65. Republic of Turkey, Ministry of Food, Agriculture and Livestock, General Directorate of Fisheries and Aquaculture.

Bayhan E., Ergin M., Temel A. et al. 2001. Sedimentology and mineralogy of surficial bottom deposits from the Aegean-Canakkale-Marmara transition (Eastern Mediterranean): Effects of marine and terrestrial factors. Mar. Geol. 175: 297-315. http://dx.doi.org/10.1016/S0025-3227(01)00142-6

Benli H.A., Cihangir B., Bizsel K.C. 1999. Investigation on demersal fishery resources in the Aegean Sea. Istanbul University Fisheries Faculty, Journal of Aquatic Products (Special Print), 301-370.

Bilgin S., Ozen O., İsmen A. et al. 2009. Bathymetric Distribution, Seasonal Growth and Mortality of the Deep-Water Rose Shrimp Parapenaeus longirostris (Decapoda: Penaeidae) in an Unexploited Stock in Saros Bay, Aegean Sea. J. Anim. Vet. Ad. 8(11): 2404-2417.

Caddy J.F. 2009. Practical issues in choosing a framework for resource assessment and management of Mediterranean and Black Sea fisheries. Medit. Mar. Sci. 10: 83-119. http://dx.doi.org/ 10.12681/mms.124

Carbonell A., Martín P., De Ranieri S. et al. 1998. Discards of the western Mediterranean trawl fleets. Rapp. Comm. int. Mer Médit. 35: 292-293

Carney R.S. 2005. Zonation of deep biota on continental margins. Oceanogr. Mar. Biol. Ann. Rev. 43: 211-278. http://dx.doi.org/10.1201/9781420037449.ch6

Cartes J.E., Sardà F. 1993. Zonation of deep-sea decapod fauna in the Catalan Sea (Western Mediterranean). Mar. Ecol. Prog. Ser. 94: $27-34$ http://dx.doi.org/10.3354/meps094027

Chilari A., Petrakis G., Evaggelos T. 2006. Aspects of the biology of the blackspot seabream (Pagellus bogaraveo) in the Ionian Sea, Greece. Fish. Res. 77: 84-91. http://dx.doi.org/10.1016/j.fishres.2005.08.003

Clarke K.R., Warwick R.M. 2001. Change in Marine Communities: An Approach to Statistical Analysis and Interpretation. 2nd ed. PRIMER-E: Plymouth.

Coker T., Turkoglu M., Satilmis H.H. 2008. Seasonal Distribution of some Fish Eggs and Larvae, and Potential Contribution of Some Large Scale Pelagic Fish Species in Saros Bay, Turkey. In Çanakkale Values Symposiums (ÇIDS), Gelibolu Bulletin Book, 27-28 August 2008, Gelibolu, Çanakkale, Turkey. 329330 pp. (in Turkish).

Coll M., Palomera I., Tudela S. et al. 2006. Trophic flows, ecosystem structure and fishing impacts in the South Catalan Sea, Northwestern Mediterranean. J. Mar. Syst. 59: 63-96. http://dx.doi.org/10.1016/j.jmarsys.2005.09.001

Damalas D., Vassilopoulou V. 2011. Chondrichthyan by-catch and 
discards in the demersal trawl fishery of the central Aegean Sea (Eastern Mediterranean). Fish. Res. 108: 142-152. http://dx.doi.org/10.1016/j.fishres.2010.12.012

Damalas D., Maravelias C.D., Katsanevakis S. et al. 2010. Seasonal abundance of non-commercial demersal fish in the eastern Mediterranean Sea in relation to hydrographic and sediment characteristics. Est. Coast. Shelf Sci. 89: 107-118. http://dx.doi.org/10.1016/j.ecss.2010.06.002

Davies R.W.D., Cripps S.J., Nickson A. et al. 2009. Defining and estimating global marine fisheries bycatch. Mar. Policy 33: 661-672. http://dx.doi.org/10.1016/j.marpol.2009.01.003

D’Onghia G., Tursi A., Maiorano P. et al. 1998. Demersal fish assemblages from the bathyal grounds of the Ionian Sea (middleeastern Mediterranean). Ital. J. Zool. 65: 287-292. http://dx.doi.org/10.1080/11250009809386834

D’Onghia G., Carlucci R., Maiorano P. et al. 2003. Discards from deep-water bottom trawling in the eastern-central Mediterranean Sea and effects of mesh size changes. J. Northwest Atl. Fish. Soc. 31: 245-262.

Duruer E.Ç., Tosunoğlu Z. 2012. Comparison of the discard characteristics of diamond and square mesh codends in the Aegean Sea trawl fishery. Ege J. Fish. Aqua. Sci. 29(1): 31-34 http://dx.doi.org/10.12714/egejfas.2012.29.1.05

Estácio S., Mendonça A., Krug H. et al. 2001. Aspects of the reproduction of six exploited demersal fish species in the Azores archipelago, Arquipélago. Life Mar. Sci. (Suppl. 2 Part B): 83-94.

European Commission 2007. Turkey 2007 Progress Report. Accompanying the Communication from the Commission to the European Parliament and the Council. Enlargement Strategy and Main Challenges 2007-2008 \{COM(2007) 663 final\}. Commission Staff Working Document. Brussels, 6.11.2007, SEC(2007) 1436, 82 pp.

European Commission 2011. Reform of the Common Fisheries Policy. Communication from the Commission to the European Parliament, the Council, the European Economic and Social Committee and the Committee of the Regions. Brussels, 13.7.2011, COM(2011) 417 final, $10 \mathrm{pp}$.

Garibaldi L., Caddy J.F. 1998. Biogeographic characterization of Mediterranean and Black Sea faunal provinces using GIS procedures. Ocean Coast. Manage. 39: 211-227. http://dx.doi.org/10.1016/S0964-5691(98)00008-8

Gislason H., Sinclair M., Sainsbury K. et al. 2000. Symposium overview: incorporating ecosystem objectives within fisheries management. ICES J. Mar. Sci. 57: 468-475. http://dx.doi.org/10.1006/jmsc. 2000.0741

Guijarro B., Massutí E. 2006. Selectivity of diamond- and squaremesh codends in the deep water crustacean trawl fishery off the Balearic Islands (western Mediterranean). ICES J. Mar. Sci. 63 : 52-67.

http://dx.doi.org/10.1016/j.icesjms.2005.08.011

Harmelin-Vivien M., Kelig M., Xavier B. et al. 2012. Possible link between prey quality, condition and growth of juvenile hake (Merluccius merluccius) in the Gulf of Lions (NW Mediterranean). Cybium 36(2): 323-328.

Ignatiades L., Psarra S., Zervakis V. et al. 2002. Phytoplankton sizebased dynamics in the Aegean Sea (Eastern Mediterranean). J. Marine Syst. 36: 11-28. http://dx.doi.org/10.1016/S0924-7963(02)00132-X

İsmen A., Özekinci U., Özen Ö. et al. 2010. Saroz Körfezi (Kuzey Ege Denizi) Demersal Balıklarının Biyo-Ekolojisi ve Populasyon Dinamiğinin Belirlenmesi Proje Raporu (Tübitak, Çaydag -106Y035). Ankara. 358 pp. (in Turkish)

Jennings S., Kaiser M.J. 1998. The effects of fishing on marine ecosystems. Ad. Mar. Biol. 34: 201-352. http://dx.doi.org/10.1016/S0065-2881(08)60212-6

Kallianiotis A., Sophronidis K, Vidoris P. et al, 2000. Demersal fish and megafaunal assemblages on the Cretan continental shelf and slope (NE Mediterranean): seasonal variation in species density, biomass diversity. Prog. Oceanog. 46: 429-455. http://dx.doi.org/10.1016/S0079-6611(00)00028-8

Kallianiotis A., Vidoris P., Sylaios G. 2004. Fish species assemblages and geographical sub-areas in the North Aegean Sea, Greece. Fish. Res. 68: 171-187. http://dx.doi.org/10.1016/j.fishres.2003.12.007

Kara O.F., Gurbet R. 1999. Investigation on industrial fishery of the Aegean Sea. The Republic of Turkey, Ministry of Agriculture and Rural Affairs, Bodrum. No: 5, 135 pp. (In Turkish)

Katsanevakis S, Maravelias, C.D., Damalas, D. et al. 2009. Spatiotemporal distribution and habitat use of commercial demer- sal species in the eastern Mediterranean Sea. Fish. Oceanogr. 18(6): 439-457.

http://dx.doi.org/10.1111/j.1365-2419.2009.00523.x

Katsanevakis S., Maravelias, C.D. 2009. Bathymetric distribution of sixteen demersal fish in the Aegean and Ionian Seas, based on generalized additive modelling. Fish. Sci. 75(1): 13-23. http://dx.doi.org/10.1007/s12562-008-0033-5

Kelleher K. 2005. Discards in the world's marine fisheries. An update. FAO Fisheries Technical Paper. No. 470. Rome, FAO. 2005. 131 pp. Includes a CD-ROM.

Keskin C., Ordines F., Guijarro B. et al. 2011a. Comparison of fish assemblages between the Sea of Marmara and the Aegean Sea (north-eastern Mediterranean). J. Mar. Biol. Assoc. U.K. 91: 1307-1318. http://dx.doi.org/10.1017/S0025315410002213

Keskin C., Turan C., Ergüden D. 2011b. Distribution of the demersal fishes on the continental shelves of the Levantine and North Aegean Seas (Eastern Mediterranean). TrJFAS 11(3): 413-423.

Klaoudatos D. Kapiris C. Conides A et al 2010. Assessment of demersal distribution between two fishing areas in North and Central Greece. Int. Aquat. Res. (Special Issue of Aqua Medit 2010 Conference, Greece) 2: 143-154

Kınacigıl H.T., İlkyaz A.T. 2012. Aegean Sea Fisheries. In: A. Tokaç, A.C. Gücü, B. Öztürk, (eds). The State of the Turkish Fisheries. Turkish Marine Research Foundation, Istanbul, Turkey. pp. 233-241.

Koral H., Oztürk H., Hanilçi N. 2008. Tectonically induced coastal uplift mechanism of Gökçeada Island, Northern Aegean Sea, Turkey. Quatern. Int http://dx.doi.org/10.1016/j.quaint.2008.04.001

Koşar I. 2009. Türkiye'de Balıçılık İstatistiklerinin İyileştirilmesi ve Avrupa Birliği Uyum Süreci. (Improvement Turkish fishery statistics and EU accession process). E.U.J. Fish. Aquat. Sci. 26 (2): 153-158.

Krug H.M. 1998. Variation in the reproductive cycle of the blackspot seabream, Pagellus bogaraveo (Brünnich, 1768) in the Azores, Arquipélago. Life Mar. Sci. 16A: 37-47

Labropoulou A., Papaconstantinou C. 2000. Community structure of deep-sea demersal fish in the North Aegean Sea (Northeastern Mediterranean). Hydrobiologia 440: 281-296.

Labropoulou A., Papaconstantinou C. 2004. Community structure and diversity of demersal fish assemblages: the role of fishery. Sci. Mar. 68(Suppl. 1): 215-226.

Labropoulou A., Papaconstantinou C. 2005. Effects of fishing on community structure of demersal fish assemblages. Bel. J. Zool. 135(2): 191-197.

Machias A., Vassilopoulou V., Vatsos D. et al. 2001. Bottom trawl discards in the northeastern Mediterranean Sea. Fish. Res. 53: 181-195. http://dx.doi.org/10.1016/S0165-7836(00)00298-8

Machias A., Maiorano P., Vassilopoulou V. et al. 2004. Sizes of discarded commercial species in the eastern central Mediterranean Sea. Fish. Res. 66: 213-222. http://dx.doi.org/10.1016/S0165-7836(03)00202-9

Maravelias C.D., Tserpes G., Pantazi M. et al. 2012. Habitat Selection and Temporal Abundance Fluctuations of Demersal Cartilaginous Species in the Aegean Sea (Eastern Mediterranean). PLoS One 7(4), e35474. http://dx.doi.org/10.1371/journal.pone.0035474

Massutí E., Reñones O., Carbonell A. et al. 1996. Demersal fish communities exploited on the continental shelf and slope off Majorca (Balearic Islands, NW Mediterranean). Vie Milieu 46: 45-55.

Massutí E., Reñones O. 2005. Demersal resource assemblages in the trawl fishing grounds of the Balearic Islands (western Mediterranean). Sci. Mar. 69(1): 167-181.

Moranta J., Stefanescu C., Massutí E. et al. 1998. Fish community structure and depth-related trends on the continental slope of the Balearic Islands (Algerian basin, western Mediterranean). Mar. Ecol. Prog. Ser. 171: 247-259. http://dx.doi.org/10.3354/meps 171247

Moranta J., Massutí E., Morales-Nin B. 2000. Fish catch composition of the deep-sea decapod crustacean fisheries in the Balearic Islands (western Mediterranean). Fish. Res. 45: 253-264. http://dx.doi.org/10.1016/S0165-7836(99)00119-8

Moranta J., Quetglas A., Massutí E. et al. 2008. Research trends on demersal fisheries oceanography in the Mediterranean. In L.P. Mertens (ed.), Biological Oceanography Research Trends. Nova Science Publishers, New York.

Ordines F., Massutí E., Guijarro B. et al. 2006. Diamond vs. square 
mesh codend in a multi-species trawl fishery of the western Mediterranean: effects on catch composition, yield, size selectivity and discards. Aquat. Living Resour. 19: 329-338. http://dx.doi.org/10.1051/alr:2007003

Özbilgin Y.D., Tosuoğlu Z., Özbilgin H. 2006. By-catch in a 40 mm PE demersal trawl codend. J. Anim. Vet. Ad. 30: 179-185.

Pazi I. 2008 Water mass properties and chemical characteristics in the Saros Gulf, Northeast Aegean Sea (Eastern Mediterranean). J. Mar. Syst. 74: 698-710. http://dx.doi.org/10.1016/j.jmarsys.2008.07.002

Petrakis G., Stergiou K.I. 1997. Size selectivity of diamond and square mesh codends for four commercial Mediterranean fish species. ICES J. Mar. Sci. 54: 13-23. http://dx.doi.org/10.1006/jmsc.1996.0172

Pielou E.C. 1969. An introduction to Mathematical Ecology. Wiley, Sons, New York.

Piet G.J., Jennings S. 2005. Response of potential fish community indicators to fishing. ICES J. Mar. Sci. 62: 214-225. http://dx.doi.org/10.1016/j.icesjms.2004.09.007

REC.CM-GFCM/33/2009/2. On the minimum mesh size in the codend of demersal trawl nets. http://151.1.154.86/gfcmwebsite/ Docs/RecRes/REC.CM-GFCM 332009 2.pdf

Sarı E., Çağatay M.N. 2001. Distributions of heavy metals in the surface sediments of the Gulf of Saros, NE Aegean Sea. Environ. Int. 26: 169-173 http://dx.doi.org/10.1016/S0160-4120(00)00097-0

Sánchez P., Demestre M., Martín P. 2004. Characterization of the discards generated by bottom trawling in the Northwestern Mediterranean. Fish. Res. 67: 71-80. http://dx.doi.org/10.1016/j.fishres.2003.08.004

Sánchez P., Sartor P., Recasens L. et al. 2007. Trawl catch composition during different fishing intensity periods in two Mediterranean demersal fishing grounds. Sci. Mar. 71(4): 765-773.

Shannon C.E., Weaver V. 1949. A mathematical theory of communication. Univ. of Illinois Press, Urbana.

Smith C.J., Papadopoulou K.N., Diliberto S. 2000. Impact of otter trawling on an eastern Mediterranean commercial trawl fishing ground. ICES J. Mar. Sci. 57: 1340-1351. http://dx.doi.org/10.1006/jmsc.2000.0927

Stefanescu C., Lloris D., Rucabado J. 1993. Deep-sea fish assemblages in the Catalan Sea (western Mediterranean) below a depth of 1000 m. Deep-Sea Res. Pt. I 40: 695-707. http://dx.doi.org/10.1016/0967-0637(93)90066-C

Stergiou K.I, Pollard D.A. 1994. A spatial analysis of the commercial fisheries catches from the Greek Aegean Sea. Fish. Res. 20: 109-135.

http://dx.doi.org/10.1016/0165-7836(94)90078-7

Stergiou K.I., Politou C.-Y., Christou E.D. et al. 1997. Selectivity experiments in the NE Mediterranean: the effect of trawl codend mesh size on species diversity and discards ICES J. Mar. Sci. 54: 774-786.

http://dx.doi.org/10.1006/jmsc.1997.0231

Stergiou K. I. Economou A., Papaconstantinou C et al. 1998. Estimates of discards in the Hellenic commercial trawl fishery. Rapp. Comm. Mer. Médit. 35: 490-491.

Tirasın E.M., Ünlüoğlu A., 2012. Demersal and Deep Water Fisheries Resources in the Turkish Seas. In A. Tokac, A.C. Gücü and B. Öztürk (eds) The State of The Turkish Fisheries. Published by Turkish Marine Research Foundation, Istanbul, Turkey. pp. $28-91$.

Tokaç A. Lök A., Tosunoğlu Z. et al. 1998. Cod-end selectivities of a modified bottom trawl for three fish species in the Aegean Sea. Fish. Res. 39: 17-31. http://dx.doi.org/10.1016/S0165-7836(98)00172-6

Tokaç A., Özbilgin H., Tosunoğlu Z. 2004. Comparison of the selectivity of PA and PE codends. Fish. Res. 67: 317-327. http://dx.doi.org/10.1016/j.fishres.2003.10.001

Tosunoğlu Z., Doğanyılmaz Y., Özbilgin Y.D. 2003a. Body shape and trawl codend selectivity for nine commercial fish species. J. Mar. Biol. Assoc. U.K. 83: 1309-1313. http://dx.doi.org/10.1017/S0025315403008737

Tosunoğlu Z., Özbilgin H., Tokaç A. 2003b. Effects of the protective bags on the cod-end selectivity in Turkish bottom trawl fishery. Arch. Fish. Mar. Res. 50: 239-252.

Tsagarakis K, Coll M., Giannoulaki M. et al. 2010. Food-web traits of the North Aegean Sea ecosystem (Eastern Mediterranean) and comparison with other Mediterranean ecosystems. Est. Coast. Shelf Sci. 88: 233-248. http://dx.doi.org/10.1016/j.ecss.2010.04.007

Tsagarakis K., Palialexis A., Vassilopoulou V. 2013. Mediterranean fishery discards: review of the existing knowledge. ICES J. Mar. Sci. http://dx.doi.org/10.1093/icesjms/fst074

Tsimenides N., Tserpes G., Machias A. et al. 1991. Distribution of fishes on the Cretan shelf. J. Fish. Biol. 39: 661-672. http://dx.doi.org/10.1111/j.1095-8649.1991.tb04396.x

Tudela S. 2004. Ecosystem effects of fishing in the Mediterranean: An analysis of the major threats of fishing gear and practices to biodiversity and marine habitats. Studies and Reviews No: 74 GFCM, FAO. Rome. 44 pp.

TUIK (Turkish Statistical Institute) 2009. Fishery Yearbook of Statistics 2009. Turkish Statistical Association. Prime Ministry, Ankara.

Vassilopoulou V., Papaconstantinou C. 1998. Discarding at sea by commercial trawlers in Greek waters. Rapp. Comm. int. Mer. Médit. 35: 502-503. 